\title{
THE EFFECT OF CURVATURE ON THE BEST CONSTANT IN THE HARDY-SOBOLEV INEQUALITIES
}

\author{
N. GHOUSSOUB AND F. ROBERT
}

\begin{abstract}
We address the question of attainability of the best constant in the following Hardy-Sobolev inequality on a smooth domain $\Omega$ of $\mathbb{R}^{n}$ :

$$
\mu_{s}(\Omega):=\inf \left\{\int_{\Omega}|\nabla u|^{2} d x ; u \in H_{1,0}^{2}(\Omega) \text { and } \int_{\Omega} \frac{|u|^{2^{\star}}}{|x|^{s}} d x=1\right\}
$$

when $0<s<2,2^{\star}:=2^{*}(s)=\frac{2(n-s)}{n-2}$, and when 0 is on the boundary $\partial \Omega$. This question is closely related to the geometry of $\partial \Omega$, as we extend here the main result obtained in 15 by proving that at least in dimension $n \geq 4$, the negativity of the mean curvature of $\partial \Omega$ at 0 is sufficient to ensure the attainability of $\mu_{s}(\Omega)$. Key ingredients in our proof are the identification of symmetries enjoyed by the extremal functions correrresponding to the best constant in half-space, as well as a fine analysis of the asymptotic behaviour of appropriate minimizing sequences. The result holds true also in dimension 3 but the more involved proof will be dealt with in a forthcoming paper [17.
\end{abstract}

\section{INTRODUCTION}

Let $\Omega$ be a smooth domain of $\mathbb{R}^{n}, n \geq 3$ and denote by $H_{1,0}^{2}(\Omega)$ the completion of $C_{c}^{\infty}(\Omega)$, the set of smooth functions compactly supported in $\Omega$, for the norm $\|u\|_{H_{1,0}^{2}(\Omega)}=\sqrt{\int_{\Omega}|\nabla u|^{2} d x}$. The Hardy-Sobolev inequality ([5], [6], [18]) asserts that for $s \in[0,2]$ and for $2^{\star}:=2^{*}(s)=\frac{2(n-s)}{n-2}$, there exists $C>0$ such that for all $u \in H_{1,0}^{2}\left(\mathbb{R}^{n}\right)$,

We define

$$
\left(\int_{\mathbb{R}^{n}} \frac{|u|^{2^{\star}}}{|x|^{s}} d x\right)^{\frac{2}{2^{\star}}} \leq C \int_{\mathbb{R}^{n}}|\nabla u|^{2} d x
$$

$$
\mu_{s}(\Omega)=\inf \left\{\frac{\int_{\Omega}|\nabla u|^{2} d x}{\left(\int_{\Omega} \frac{|u|^{\star}}{|x|^{s}} d x\right)^{\frac{2}{2^{\star}}}} ; u \in H_{1,0}^{2}(\Omega) \backslash\{0\}\right\},
$$

and we consider the corresponding ground state solutions in $H_{1,0}^{2}(\Omega) \cap C^{1}(\bar{\Omega})$ for

$$
\begin{cases}\Delta u=\frac{u^{2^{\star}-1}}{|x|^{s}} & \text { in } \mathcal{D}^{\prime}(\Omega) \\ u>0 & \text { in } \Omega \\ u=0 & \text { on } \partial \Omega .\end{cases}
$$

Date: February 25th 2005.

Research partially supported by the Natural Sciences and Engineering Research Council of Canada. The first named author gratefully acknowledges the hospitality and support of the Université de Nice where this work was initiated.

The second named author gratefully acknowledges the hospitality and support of the University of British Columbia where this work was completed. 
where here and throughout the paper, $\Delta=-\sum_{i} \partial_{i i}$ is the Laplacian with minus sign convention.

It is well known (see for instance [27]) that in the non-singular case i.e., when $s=0$, we have $\mu_{s}(\Omega)=\mu_{s}\left(\mathbb{R}^{n}\right)$ for any domain $\Omega$ and that $\mu_{s}(\Omega)$ is never attained unless $\operatorname{cap}\left(\mathbb{R}^{n} \backslash \Omega\right)=0$. In this situation, the limiting spaces after blow-up of solutions of (3) is $\mathbb{R}^{n}$. It was shown in 18 that the same result holds true for any $0<s<2$ as long as 0 belongs to the interior of a domain.

However, the fact that things may be different when $0 \in \partial \Omega$ first emerged in a paper by Egnell [12] where he considers open cones of the form $C=\left\{x \in \mathbb{R}^{n} ; x=\right.$ $r \theta, \theta \in D$ and $r>0\}$ where the base $D$ is a connected domain of the unit sphere $S^{n-1}$ of $\mathbb{R}^{n}$. Egnell showed that $\mu_{s}(C)$ is then attained for $0<s<2$ even when $\bar{C} \neq \mathbb{R}^{n}$. This obviously applies to a half-space $\mathbb{R}_{-}^{n}=\left\{x \in \mathbb{R}_{-}^{n} / x_{1}<0\right\}$, where $x_{1}$ denotes the first coordinate of a generic point $x \in \mathbb{R}^{n}$ in the canonical basis of $\mathbb{R}^{n}$.

Half-spaces containing 0 on their boundary were identified in [15] as the limiting spaces after blow-up in the case where $\partial \Omega$ is smooth at 0 , and the curvature of the boundary at 0 then gets to play an important role. In our context, we specify the orientation of $\partial \Omega$ in such a way that the normal vectors of $\partial \Omega$ are pointing outward from the domain $\Omega$. It was shown in 15 that in dimension $n \geq 4$, the negativity of all principal curvatures at 0 -which is essentially a condition of "strict concavity" at 0- leads to attainability of the best constant for problems with Dirichlet boundary conditions, while the Neumann problems required the positivity of the mean curvature at 0 . On the other hand, standard Pohozaev type arguments show non-attainability in the cases where $\Omega$ is convex or star-shaped at 0 .

In this paper, we improve and complete the results in [15] in a substantial way by showing that for the best constant to be achieved, it is sufficient that the mean curvature be negative. This is now quite similar but dual to the case with Neumann boundary conditions which requires the mean curvature to be positive.

More precisely, assume that the principal curvatures $\alpha_{1}, \ldots, \alpha_{n-1}$ of $\partial \Omega$ at 0 are finite. The oriented boundary $\partial \Omega$ near the origin can then be represented (up to rotating the coordinates if necessary) by $x_{1}=\varphi_{0}\left(x^{\prime}\right)=-\frac{1}{2} \sum_{i=2}^{n} \alpha_{i-1} x_{i}^{2}+o\left(\left|x^{\prime}\right|^{2}\right)$, where $x^{\prime}=\left(x_{2}, \ldots, x_{n}\right) \in B_{\delta}(0) \cap\left\{x_{1}=0\right\}$ for some $\delta>0$ and where $B_{\delta}(0)$ is the ball in $\mathbb{R}^{n}$ centered at 0 with radius $\delta$. If one assumes the principal curvatures at 0 to be negative, that is if

$$
\max _{1 \leq i \leq n-1} \alpha_{i}<0
$$

then the sectional curvature at 0 is negative and therefore $\partial \Omega$-viewed as an $(n-1)$ Riemannian submanifold of $\mathbb{R}^{n}$ - is strictly convex at 0 (see for instance [14]). The latter property means that there exists a neighborhood $U$ of 0 in $\partial \Omega$, such that the whole of $U$ lies on one side of a hyperplane $H$ that is tangent to $\partial \Omega$ at 0 and $U \cap H=\{0\}$, and so does the complementary $\mathbb{R}^{n} \backslash \Omega$, at least locally. The above curvature condition then amounts to a notion of strict local convexity of $\mathbb{R}^{n} \backslash \Omega$ at 0 . Our main result below shows that at least for dimension greater than 4 , it is sufficient to assume that

$$
\sum_{1 \leq i \leq n-1} \alpha_{i}<0
$$

Theorem 1.1. Let $\Omega$ be a smooth bounded oriented domain of $\mathbb{R}^{n}$ where $n \geq 4$, such that $0 \in \partial \Omega$ and assume $s \in(0,2)$. If the mean curvature of $\partial \Omega$ at 0 is negative, then the infimum $\mu_{s}(\Omega)$ in (2) is achieved. In addition, the set of minimizers of (2) is pre-compact in the $H_{1,0}^{2}(\Omega)$-topology. 
The first difficulty we have to face here is that the extremals for (2) when $\Omega=\mathbb{R}_{-}^{n}$ are not known explicitely, and our first result below -proved in section 2 - is the identification of certain symmetries enjoyed by these extremals -and actually all positive solutions- on half-space.

Theorem 1.2. Let $n \geq 3, s \in(0,2)$ and consider $u \in C^{2}\left(\mathbb{R}_{-}^{n}\right) \cap C^{1}\left(\overline{\mathbb{R}_{-}^{n}}\right)$ such that

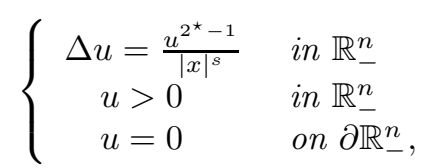

where $2^{\star}=\frac{2(n-s)}{n-2}$. Assume that for some $C>0, u(x) \leq C(1+|x|)^{1-n}$ for all $x \in \mathbb{R}_{-}^{n}$. Then we have that $u \circ \sigma=u$ for all isometry of $\mathbb{R}^{n}$ such that $\sigma\left(\mathbb{R}_{-}^{n}\right)=\mathbb{R}_{-}^{n}$. In particular, there exists $v \in C^{2}\left(\mathbb{R}_{-}^{\star} \times \mathbb{R}\right) \cap C^{1}\left(\mathbb{R}_{-} \times \mathbb{R}\right)$ such that for all $x_{1}<0$ and all $x^{\prime} \in \mathbb{R}^{n-1}$, we have that $u\left(x_{1}, x^{\prime}\right)=v\left(x_{1},\left|x^{\prime}\right|\right)$.

The attainability result is then obtained by combining this new information with a fine study of the asymptotic behaviour of solutions to the corresponding subcritical pde's. They can eventually develop a singularity at zero as we approach the critical exponent $2^{*}(s)$, and for that we proceed to completely describe the way they may blow up, which makes for an interesting analysis in its own right.

Indeed, assume $\Omega$ is a smooth bounded domain of $\mathbb{R}^{n}$ such that $0 \in \partial \Omega$ and consider for any $\epsilon \in\left(0,2^{\star}-2\right)$, the infimum

$$
\mu_{s}^{\epsilon}(\Omega):=\inf _{u \in H_{1,0}^{2}(\Omega) \backslash\{0\}} \frac{\int_{\Omega}|\nabla u|^{2} d x}{\left(\int_{\Omega} \frac{|u|^{2^{\star}-\epsilon}}{|x|^{s}} d x\right)^{\frac{2}{2^{\star}-\epsilon}}},
$$

which is achieved by a function $u_{\epsilon} \in H_{1,0}^{2}(\Omega), u_{\epsilon}>0$ in $\Omega$ in $C^{1}(\bar{\Omega}) \cap C^{2}(\bar{\Omega} \backslash\{0\})$ that satisfies the system

$$
\begin{cases}\Delta u_{\epsilon}=\frac{u_{\epsilon}^{2^{\star}-1-\epsilon}}{|x|^{s}} & \text { in } \mathcal{D}^{\prime}(\Omega) \\ u_{\epsilon}>0 & \text { in } \Omega \\ \int_{\Omega} \frac{\left|u_{\epsilon}\right|^{2^{\star}}-\epsilon}{|x|^{s}} d x=\left(\mu_{s}^{\epsilon}(\Omega)\right)^{\frac{2^{\star}-\epsilon}{2^{\star}-2-\epsilon}} & \end{cases}
$$

The bulk of the paper (beyond section 2) consists of proving the following estimate.

Theorem 1.3. Let $\Omega$ be a smooth bounded oriented domain of $\mathbb{R}^{n}$ where $n \geq 4$, and assuming that $u_{\epsilon}$ converges weakly to zero (i.e. when blow-up occurs), then there exists $v$ solution for (4) such that

$$
\int_{\mathbb{R}_{-}^{n}}|\nabla v|^{2} d x=\mu_{s}(\Omega)^{\frac{2^{\star}}{2^{\star}-2}}=\mu_{s}\left(\mathbb{R}_{-}^{n}\right)^{\frac{2^{\star}}{2^{\star}-2}},
$$

while -modulo passing to a subsequence- we have

$$
\lim _{\epsilon \rightarrow 0} \epsilon\left(\max _{\Omega} u_{\epsilon}\right)^{\frac{2}{n-2}}=\frac{(n-s) \int_{\partial \mathbb{R}_{-}^{n}}|x|^{2}|\nabla v|^{2} d x}{n(n-2)^{2} \mu_{s}\left(\mathbb{R}_{-}^{n}\right)^{\frac{n-s}{2-s}}} \cdot H(0) .
$$

where $H(0)$ is the mean curvature of the oriented boundary $\partial \Omega$ at 0 .

These techniques actually allow us to prove the following existence theorem. We shall say that a function is in $C^{1}(\bar{\Omega})$ if it can be extended to a $C^{1}$-function in a neighborhood of $\Omega$. 
Theorem 1.4. Let $\Omega$ be a smooth bounded oriented domain of $\mathbb{R}^{n}$ where $n \geq 4$, such that $0 \in \partial \Omega$. Assume $s \in(0,2)$ and consider $a \in C^{1}(\bar{\Omega})$ such that the operator $\Delta+a$ is coercive in $\Omega$. If the mean curvature of $\partial \Omega$ at 0 is negative, then there exists a solution $u \in H_{1,0}^{2}(\Omega) \cap C^{1}(\bar{\Omega})$ for

$$
\begin{cases}\Delta u+a u=\frac{u^{2^{\star}-1}}{|x|^{s}} & \text { in } \mathcal{D}^{\prime}(\Omega) \\ u>0 & \text { in } \Omega \\ u=0 & \text { on } \partial \Omega .\end{cases}
$$

The study of blow-up solutions in certain nonlinear elliptic equations was initiated by Atkinson-Peletier [1] (see also Brézis-Peletier 3]). In the Riemannian context, such asymptotics were first studied by Schoen [28] and Hebey-Vaugon [23]. The techniques of blow-up have been developed in a general context by Druet, Hebey and the second author [10]. They turned out to be very powerful tools for the study of best constant problems in Sobolev inequalities, see for instance Druet 7], Hebey-Vaugon [23, 24] and Robert [26]). We also mention the work of Han [21], Hebey [22, Druet-Robert [1] and Robert 25]) on the asymptotics for solutions to nonlinear pde's, the 3-dimensional conjecture of Brézis solved by Druet [8] and the intricate compactness issues in the Riemannian context (see for instance Schoen 28 and Druet 9]).

In a forthcoming paper [16, we shall establish a more refined compactness result which yields an infinite number of sign changing solutions for (3). In another forthcoming article [17, we tackle similar questions for various critical equations involving a whole affine subspace of singularities on the boundary.

\section{Symmetry of the Positive solutions to The Limit EQUATION}

This section is devoted to the proof of Theorem 1.2 that is the symmetry property for the positive solutions to the limit equation on $\mathbb{R}_{-}^{n}$. For that, we consider $u \in C^{2}\left(\mathbb{R}_{-}^{n}\right) \cap C^{1}\left(\overline{\mathbb{R}_{-}^{n}}\right)$ that verifies the system (4) while verifying for some $C>0$ the bound

$$
u(x) \leq \frac{C}{(1+|x|)^{n-1}}
$$

for all $x \in \mathbb{R}_{-}^{n}$. Denoting by $\vec{e}_{1}$ the first vector of the canonical basis of $\mathbb{R}^{n}$, we consider the open ball $D:=B_{1 / 2}\left(-\frac{1}{2} \vec{e}_{1}\right)$ and define

$$
v(x):=|x|^{2-n} u\left(\vec{e}_{1}+\frac{x}{|x|^{2}}\right)
$$

for all $x \in \bar{D} \backslash\{0\}$ and $v(0)=0$. Clearly, this is well-defined.

Step 2.1: We claim that

$$
v \in C^{2}(D) \cap C^{1}(\bar{D}) \text { and } \frac{\partial v}{\partial \nu}<0 \text { on } \partial D
$$

where $\partial / \partial \nu$ denotes the outward normal derivative.

Proof. It follows from the assumptions on $u$ that $v \in C^{2}(D) \cap C^{1}(\bar{D} \backslash\{0\})$. Moreover, $v(x)>0$ for all $x \in D$ and $v(x)=0$ for all $x \in \partial D \backslash\{0\}$. It follows from (5) that there exists $C>0$ such that

$$
v(x) \leq C|x|
$$


for all $x \in \bar{D} \backslash\{0\}$. Since $v(0)=0$, we have that $v \in C^{0}(\bar{D})$. The function $v$ verifies the equation

$$
\Delta v=\frac{v^{2^{\star}-1}}{\left.\left.|x+| x\right|^{2} \vec{e}_{1}\right|^{s}}=\frac{v^{2^{\star}-1}}{|x|^{s}\left|x+\vec{e}_{1}\right|^{s}}
$$

in $D$. Since $-\vec{e}_{1} \in \partial D \backslash\{0\}$ and $v \in C^{1}(\bar{D} \backslash\{0\}) \cap C^{0}(\bar{D})$, there exists $C>0$ such that

$$
v(x) \leq C\left|x+\vec{e}_{1}\right|
$$

for all $x \in \bar{D}$. It then follows from (87), (9), (10) and standard elliptic theory that $v \in C^{1}(\bar{D})$. Since $v>0$ in $D$, it follows from Hopf's Lemma that $\frac{\partial v}{\partial \nu}<0$ on $\partial D$.

We prove the symmetry of $u$ by proving a symmetry property of $v$, which is defined on a ball. Our proof uses the moving plane method. We take largely inspiration in [19] and [4. Classically, for any $\mu \geq 0$ and any $x=\left(x^{\prime}, x_{n}\right) \in \mathbb{R}^{n}$ $\left(x^{\prime} \in \mathbb{R}^{n-1}\right.$ and $\left.x_{n} \in \mathbb{R}\right)$, we let

$$
x_{\mu}=\left(x^{\prime}, 2 \mu-x_{n}\right) \text { and } D_{\mu}=\left\{x \in D / x_{\mu} \in D\right\} .
$$

It follows from Hopf's Lemma (See (7)) that there exists $\epsilon_{0}>0$ such that for any $\mu \in\left(\frac{1}{2}-\epsilon_{0}, \frac{1}{2}\right)$, we have that $D_{\mu} \neq \emptyset$ and $v(x) \geq v\left(x_{\mu}\right)$ for all $x \in D_{\mu}$ such that $x_{n} \leq \mu$. We let $\mu \geq 0$. We say that $\left(P_{\mu}\right)$ holds if:

$$
D_{\mu} \neq \emptyset \text { and } v(x) \geq v\left(x_{\mu}\right) \text { for all } x \in D_{\mu} \text { such that } x_{n} \leq \mu \text {. }
$$

We let

$$
\lambda:=\min \left\{\mu \geq 0 ;\left(P_{\nu}\right) \text { holds for all } \nu \in\left(\mu, \frac{1}{2}\right)\right\} .
$$

Step 2.2: We claim that $\lambda=0$.

Proof. We proceed by contradiction and assume that $\lambda>0$. We then get that $D_{\lambda} \neq \emptyset$ and that $\left(P_{\lambda}\right)$ holds. We let

$$
w(x):=v(x)-v\left(x_{\lambda}\right)
$$

for all $x \in D_{\lambda} \cap\left\{x_{n}<\lambda\right\}$. Since $\left(P_{\lambda}\right)$ holds, we have that $w(x) \geq 0$ for all $x \in D_{\lambda} \cap\left\{x_{n}<\lambda\right\}$. With the equation (9) of $v$ and $\left(P_{\lambda}\right)$, we get that

$$
\begin{aligned}
\Delta w & =\frac{v(x)^{2^{\star}-1}}{\left.\left.|x+| x\right|^{2} \vec{e}_{1}\right|^{s}}-\frac{v\left(x_{\lambda}\right)^{2^{\star}-1}}{\left.\left.\left|x_{\lambda}+\right| x_{\lambda}\right|^{2} \vec{e}_{1}\right|^{s}} \\
& \geq v\left(x_{\lambda}\right)^{2^{\star}-1}\left(\frac{1}{\left.\left.|x+| x\right|^{2} \vec{e}_{1}\right|^{s}}-\frac{1}{\left.\left.\left|x_{\lambda}+\right| x_{\lambda}\right|^{2} \vec{e}_{1}\right|^{s}}\right)
\end{aligned}
$$

for all $x \in D_{\lambda} \cap\left\{x_{n}<\lambda\right\}$. With straightforward computations, we have that

$$
\begin{aligned}
& \left|x_{\lambda}\right|^{2}-|x|^{2}=4 \lambda\left(\lambda-x_{n}\right) \\
& \left.\left.\left.\left|x_{\lambda}+\right| x_{\lambda}\right|^{2} \vec{e}_{1}\right|^{2}-\left.\left.|x+| x\right|^{2} \vec{e}_{1}\right|^{2}=\left(\left|x_{\lambda}\right|^{2}-|x|^{2}\right)\left(1+\left|x_{\lambda}\right|^{2}+|x|^{2}+2 x_{1}\right)\right)
\end{aligned}
$$

for all $x \in \mathbb{R}^{n}$. It follows that $\Delta w(x)>0$ for all $x \in D_{\lambda} \cap\left\{x_{n}<\lambda\right\}$. Note that we have used that $\lambda>0$. It then follows from Hopf's Lemma and the strong comparison principle that

$$
w>0 \text { in } D_{\lambda} \cap\left\{x_{n}<\lambda\right\} \text { and } \frac{\partial w}{\partial \nu}<0 \text { on } D_{\lambda} \cap\left\{x_{n}=\lambda\right\} .
$$


By definition, there exists a sequence $\left(\lambda_{i}\right)_{i \in \mathbb{N}} \in \mathbb{R}$ and a sequence $\left(x^{i}\right)_{i \in \mathbb{N}} \in D$ such that $\lambda_{i}<\lambda, x^{i} \in D_{\lambda_{i}},\left(x^{i}\right)_{n}<\lambda_{i}, \lim _{i \rightarrow+\infty} \lambda_{i}=\lambda$ and

$$
v\left(x^{i}\right)<v\left(\left(x^{i}\right)_{\lambda_{i}}\right)
$$

for all $i \in \mathbb{N}$. Up to extraction a subsequence, we assume that there exists $x \in$ $\bar{D}_{\lambda} \cap\left\{x_{n} \leq \lambda\right\}$ such that $\lim _{i \rightarrow+\infty} x^{i}=x$ with $x_{n} \leq \lambda$. Passing to the limit $i \rightarrow+\infty$ in (13), we get that $v(x) \leq v\left(x_{\lambda}\right)$. It follows from this last inequality and (12) that $v(x)-v\left(x_{\lambda}\right)=w(x)=0$, and then $x \in \partial\left(D_{\lambda} \cap\left\{x_{n}<\lambda\right\}\right)$.

Case 1: If $x \in \partial D$. Then $v\left(x_{\lambda}\right)=0$ and $x_{\lambda} \in \partial D$. Since $D$ is a ball and $\lambda>0$, we get that $x=x_{\lambda} \in \partial D$. Since $v$ is $C^{1}$, we get that there exists $\tau_{i} \in\left(\left(x^{i}\right)_{n}, 2 \lambda_{i}-\left(x^{i}\right)_{n}\right)$ such that

$$
v\left(x^{i}\right)-v\left(\left(x^{i}\right)_{\lambda_{i}}\right)=\partial_{n} v\left(\left(x^{\prime}\right)^{i}, \tau_{i}\right) \times 2\left(\left(x^{i}\right)_{n}-\lambda_{i}\right)
$$

Letting $i \rightarrow+\infty$, using that $\left(x^{i}\right)_{n}<\lambda_{i}$ and (13), we get that $\partial_{n} v(x) \geq 0$. On the other hand, we have that

$$
\partial_{n} v(x)=\frac{\partial v}{\partial \nu}(x) \cdot\left(\nu(x) \mid \vec{e}_{n}\right)=\frac{\lambda}{\left|x+\vec{e}_{1} / 2\right|} \frac{\partial v}{\partial \nu}(x)<0 .
$$

A contradiction with (7).

Case 2: If $x \in D$. Since $v\left(x_{\lambda}\right)=v(x)$, we then get that $x_{\lambda} \in D$. Since $x \in$ $\partial\left(D_{\lambda} \cap\left\{x_{n}<\lambda\right\}\right)$, we then get that $x \in D \cap\left\{x_{n}=\lambda\right\}$. With the same argument as in the preceding step, we get that $\partial_{n} v(x) \geq 0$. On the other hand, with (12), we get that $2 \partial_{n} v(x)=\partial_{n} w(x)<0$. A contradiction.

In all the cases, we have obtained a contradiction. This proves that $\lambda=0$.

Step 2.3: Here goes the final argument. Since $\lambda=0$, it follows from the definition (11) of $\lambda$ that $v\left(x^{\prime}, x_{n}\right) \geq v\left(x^{\prime},-x_{n}\right)$ for all $x \in D$ such that $x_{n} \leq 0$. With the same technique, we get the reverse inequality, and then, we get that

$$
v\left(x^{\prime}, x_{n}\right)=v\left(x^{\prime},-x_{n}\right)
$$

for all $x=\left(x^{\prime}, x_{n}\right) \in D$. In other words, $v$ is symmetric with respect to the hyperplane $\left\{x_{n}=0\right\}$. The same analysis holds for any hyperplane containing $\overrightarrow{e_{1}}$. Coming back to the initial function $u$, this complete the proof of Theorem 1.2.

\section{Test-FunCtions estimates}

We first introduce some definitions and notations. We consider a family $\left(a_{\epsilon}\right)_{\epsilon>0} \in$ $C^{1}(\Omega)$ and a function $a \in C^{1}(\Omega)$ such that there exists an open subset $\mathcal{U} \subset \mathbb{R}^{n}$ such that $a_{\epsilon}, a$ can be extended to $\mathcal{U}$ by $C^{1}$-functions that we still denote by $a_{\epsilon}, a$. We assume that they satisfy

$$
\bar{\Omega} \subset \subset \mathcal{U} \text { and } \lim _{\epsilon \rightarrow 0} a_{\epsilon}=a \text { in } C_{l o c}^{1}(\mathcal{U}) .
$$

We assume that

$$
\Delta+a \text { is coercive in } \Omega,
$$

that is, there exists $c_{0}>0$ such that

$$
\int_{\Omega}\left(|\nabla \varphi|^{2}+a \varphi^{2}\right) d x \geq c_{0} \int_{\Omega} \varphi^{2} d x
$$


for all $\varphi \in C_{c}^{1}(\Omega)$, the set of $C^{1}$-functions compactly supported in $\Omega$. Finally, we let

$$
\mu_{s, a}(\Omega)=\inf \frac{\int_{\Omega}\left(|\nabla u|^{2}+a u^{2}\right) d x}{\left(\int_{\Omega} \frac{|u|^{2^{\star}}}{|x|^{s}} d x\right)^{\frac{2}{2^{\star}}}} .
$$

Note that $\mu_{s, 0}(\Omega)=\mu_{s}(\Omega)$. We let $x_{0} \in \partial \Omega$. Since $\partial \Omega$ is smooth and $x_{0} \in \partial \Omega$, there exist $U, V$ open subsets of $\mathbb{R}^{n}$, there exists $I$ an open intervall of $\mathbb{R}$, there exists $U^{\prime}$ an open subset of $\mathbb{R}^{n-1}$ such that $0 \in U=I \times U^{\prime}$ and $x_{0} \in V$. There exist $\varphi \in C^{\infty}(U, V)$ and $\varphi_{0} \in C^{\infty}\left(U^{\prime}\right)$ such that

(i) $\quad \varphi: U \rightarrow V$ is a $C^{\infty}$ - diffeomorphism

(ii) $\varphi(0)=x_{0}$

(iii) $\quad D_{0} \varphi=I d_{\mathbb{R}^{n}}$

(iv) $\varphi\left(U \cap\left\{x_{1}<0\right\}\right)=\varphi(U) \cap \Omega$ and $\varphi\left(U \cap\left\{x_{1}=0\right\}\right)=\varphi(U) \cap \partial \Omega$.

(v) $\varphi\left(x_{1}, y\right)=x_{0}+\left(x_{1}+\varphi_{0}(y), y\right)$ for all $\left(x_{1}, y\right) \in I \times U^{\prime}=U$

(vi) $\varphi_{0}(0)=0$ and $\nabla \varphi_{0}(0)=0$.

Here $D_{x} \varphi$ denotes the differential of $\varphi$ at $x$. This chart will be useful throughout all the paper.

The first result we prove is an upper bound for $\mu_{s, a}(\Omega)$.

Proposition 3.1. Let $\Omega$ be a smooth bounded domain of $\mathbb{R}^{n}, n \geq 3$, such that $0 \in \partial \Omega$. If $a \in C^{0}(\bar{\Omega})$ and $s \in(0,2)$, then $\mu_{s, a}(\Omega) \leq \mu_{s}\left(\mathbb{R}_{-}^{n}\right)$.

Proof. Let $\alpha>0$ and $u \in C_{c}^{\infty}\left(\mathbb{R}_{-}^{n}\right) \backslash\{0\}$ such that

$$
\frac{\int_{\mathbb{R}_{-}^{n}}|\nabla u|^{2} d x}{\left(\int_{\mathbb{R}_{-}^{n}} \frac{|u|^{\star}}{|x|^{s}} d x\right)^{\frac{2}{2^{\star}}}} \leq \mu_{s}\left(\mathbb{R}_{-}^{n}\right)+\alpha .
$$

Taking $x_{0}=0$ in (16), we define

$$
u_{\epsilon}(x)=\epsilon^{-\frac{n-2}{2}} u\left(\frac{\varphi^{-1}(x)}{\epsilon}\right)
$$

for all $x \in \Omega$ and all $\epsilon>0$. As easily checked, for $\epsilon>0$ small enough, we have that

$$
u_{\epsilon} \in C_{c}^{\infty}(\Omega) \text {. }
$$

With a change of variable, we get that

$$
\int_{\Omega} \frac{\left|u_{\epsilon}\right|^{2^{\star}}}{|x|^{s}} d x=\int_{\mathbb{R}_{-}^{n}} \frac{|u(y)|^{2^{\star}}}{\left|\frac{\varphi(\epsilon y)}{\epsilon}\right|^{s}} \cdot|\operatorname{Jac}(\epsilon y)| d y
$$

Since $u$ is compactly supported, we get with point (iii) of (16) and Lebesgue's convergence theorem that

$$
\lim _{\epsilon \rightarrow 0} \int_{\Omega} \frac{\left|u_{\epsilon}\right|^{2^{\star}}}{|x|^{s}} d x=\int_{\mathbb{R}_{-}^{n}} \frac{|u|^{2^{\star}}}{|x|^{s}} d x .
$$

On the other hand, we have that

$$
\int_{\Omega}\left(\left|\nabla u_{\epsilon}\right|^{2}+a u_{\epsilon}^{2}\right) d x=\int_{\mathbb{R}_{-}^{n}}\left(|\nabla u|_{g_{\epsilon}}^{2}+\epsilon^{2} a \circ \varphi(\epsilon x) u^{2}\right) \cdot \sqrt{\left|g_{\epsilon}\right|} d x,
$$


where $\left(g_{\epsilon}(x)\right)_{i j}=\left(\partial_{i} \varphi(\epsilon x), \partial_{j} \varphi(\epsilon x)\right)$, and $\left|g_{\epsilon}\right|=\operatorname{det}\left(g_{\epsilon}\right)$. With point (iii) of (16) and Lebesgue's convergence theorem, we get that

$$
\lim _{\epsilon \rightarrow 0} \int_{\Omega}\left(\left|\nabla u_{\epsilon}\right|^{2}+a u_{\epsilon}^{2}\right) d x=\int_{\mathbb{R}_{-}^{n}}|\nabla u|^{2} d x .
$$

As a consequence, we get that

$$
\mu_{s, a}(\Omega) \leq \frac{\int_{\Omega}\left(\left|\nabla u_{\epsilon}\right|^{2}+a u_{\epsilon}^{2}\right) d x}{\left(\int_{\Omega} \frac{\left|u_{\epsilon}\right|^{2^{\star}}}{|x|^{s}} d x\right)^{\frac{2}{2^{\star}}}}=\frac{\int_{\mathbb{R}_{-}^{n}}|\nabla u|^{2} d x}{\left(\int_{\mathbb{R}_{-}^{n}} \frac{|u|^{2^{\star}}}{|x|^{s}} d x\right)^{\frac{2}{2^{\star}}}}+o(1) \leq \mu_{s}\left(\mathbb{R}_{-}^{n}\right)+\alpha+o(1)
$$

where $\lim _{\epsilon \rightarrow 0} o(1)=0$. Letting $\epsilon \rightarrow 0$ and $\alpha \rightarrow 0$ yields the conclusion of the proposition.

\section{The SubCritical CASE}

Step 4.1: In order to construct minimizers for $\mu_{s, a}(\Omega)$, we consider a subcritical minimization problem for which we recover compactness. This is the object of the following proposition.

Proposition 4.1. Let $\Omega$ be a smooth bounded domain of $\mathbb{R}^{n}, n \geq 3$ and $s \in(0,2)$. For any $\epsilon \in\left(0,2^{\star}-2\right)$, we let $a_{\epsilon} \in C^{1}(\bar{\Omega})$ such that $\Delta+a_{\epsilon}$ is coercive. Then for any $\epsilon \in\left(0,2^{\star}-2\right)$, the infimum

$$
\mu_{s, a_{\epsilon}}^{\epsilon}(\Omega):=\inf _{u \in H_{1,0}^{2}(\Omega) \backslash\{0\}} \frac{\int_{\Omega}\left(|\nabla u|^{2}+a_{\epsilon} u^{2}\right) d x}{\left(\int_{\Omega} \frac{|u|^{2^{\star}}-\epsilon}{|x|^{s}} d x\right)^{\frac{2}{2^{\star}-\epsilon}}},
$$

is achieved by a function $u_{\epsilon} \in H_{1,0}^{2}(\Omega), u_{\epsilon}>0$ in $\Omega$. Moreover, $u_{\epsilon} \in C^{1}(\bar{\Omega}) \cap$ $C^{2}(\bar{\Omega} \backslash\{0\})$ and can be assumed to satisfy the system

$$
\begin{cases}\Delta u_{\epsilon}+a_{\epsilon} u_{\epsilon}=\frac{u_{\epsilon}^{2^{\star}-1-\epsilon}}{|x|^{s}} & \text { in } \mathcal{D}^{\prime}(\Omega) \\ u_{\epsilon}>0 & \text { in } \Omega \\ \int_{\Omega} \frac{|u|^{2^{\star}-\epsilon}}{|x|^{s}} d x=\left(\mu_{s, a_{\epsilon}}^{\epsilon}(\Omega)\right)^{\frac{2^{\star}-\epsilon}{2^{2}-2-\epsilon}} & \end{cases}
$$

Proof. This result is quite standard. We prove the proposition for the sake of completeness. We claim that there exists a minimizer for $\mu_{s, a_{\epsilon}}^{\epsilon}(\Omega)$. Indeed, let $\left(u_{k}\right)_{k \in \mathbb{N}} \in H_{1,0}^{2}(\Omega)$ be a minimizing sequence for $\mu_{s, a_{\epsilon}}^{\epsilon}(\Omega)$ such that

$$
\int_{\Omega} \frac{\left|u_{k}\right|^{2^{\star}-\epsilon}}{|x|^{s}} d x=1 \text { and } \mu_{s, a_{\epsilon}}^{\epsilon}(\Omega)=\int_{\Omega}\left(\left|\nabla u_{k}\right|^{2}+a_{\epsilon} u_{k}^{2}\right) d x+o(1)
$$

where $\lim _{k \rightarrow+\infty} o(1)=0$. Since $\left\|u_{k}\right\|_{H_{1,0}^{2}(\Omega)}=O(1)$ when $k \rightarrow+\infty$, there exists $\tilde{u}_{\epsilon} \in H_{1,0}^{2}(\Omega)$ such that, up to a subsequence, $u_{k} \rightarrow \tilde{u}_{\epsilon}$ weakly in $H_{1,0}^{2}(\Omega)$ when $k \rightarrow+\infty$ and $\lim _{k \rightarrow+\infty} u_{k}(x)=\tilde{u}_{\epsilon}(x)$ a.e. in $\Omega$. Let $\theta_{k}=u_{k}-\tilde{u}_{\epsilon} \in H_{1,0}^{2}(\Omega)$. As easily checked, we have that

$$
\mu_{s, a_{\epsilon}}^{\epsilon}(\Omega)=\int_{\Omega}\left(\left|\nabla \tilde{u}_{\epsilon}\right|^{2}+a_{\epsilon} \tilde{u}_{\epsilon}^{2}\right) d x+\int_{\Omega}\left|\nabla \theta_{k}\right|^{2} d x+o(1),
$$


where $\lim _{k \rightarrow+\infty} o(1)=0$. Let $\eta \in C_{c}^{\infty}(\mathbb{R})$ such that $\eta(x)=1$ for all $x \in[-1,1]$. Let $A>0$. With Lebesgue's theorem, we have that

$$
\begin{aligned}
& \left|\int_{\Omega} \frac{\left|u_{k}\right|^{2^{\star}-\epsilon}}{|x|^{s}} d x-\int_{\Omega} \frac{\left|\tilde{u}_{\epsilon}\right|^{2^{\star}-\epsilon}}{|x|^{s}} d x\right| \\
& =\left|\int_{\Omega}\left(\eta\left(\frac{u_{k}}{A}\right) \frac{\left|u_{k}\right|^{2^{\star}}-\epsilon}{|x|^{s}}-\eta\left(\frac{\tilde{u}_{\epsilon}}{A}\right) \frac{\left|\tilde{u}_{\epsilon}\right|^{2^{\star}-\epsilon}}{|x|^{s}}\right) d x\right| \\
& +\int_{\Omega}\left|1-\eta\left(\frac{u_{k}}{A}\right)\right| \frac{\left|u_{k}\right|^{2^{\star}-\epsilon}}{|x|^{s}} d x+\int_{\Omega}\left|1-\eta\left(\frac{\tilde{u}_{\epsilon}}{A}\right)\right| \frac{\left|\tilde{u}_{\epsilon}\right|^{2^{\star}-\epsilon}}{|x|^{s}} d x \\
& \leq o(1)+\frac{1}{A^{\epsilon}} \int_{\Omega}\left|1-\eta\left(\frac{u_{k}}{A}\right)\right| \frac{\left|u_{k}\right|^{2^{\star}}}{|x|^{s}} d x+\frac{1}{A^{\epsilon}} \int_{\Omega}\left|1-\eta\left(\frac{\tilde{u}_{\epsilon}}{A}\right)\right| \frac{\left|\tilde{u}_{\epsilon}\right|^{2^{\star}}}{|x|^{s}} d x \\
& \leq o(1)+\frac{1}{A^{\epsilon}} \int_{\Omega} \frac{\left|u_{k}\right|^{2^{\star}}}{|x|^{s}} d x+\frac{1}{A^{\epsilon}} \int_{\Omega} \frac{\left|\tilde{u}_{\epsilon}\right|^{2^{\star}}}{|x|^{s}} d x \\
& \leq o(1)+\frac{1}{A^{\epsilon}} \mu_{s}(\Omega)^{-\frac{2^{\star}}{2}}\left(\left\|u_{k}\right\|_{H_{1,0}^{2}(\Omega)}^{2^{\star}}+\left\|\tilde{u}_{\epsilon}\right\|_{H_{1,0}^{2}(\Omega)}^{2^{\star}}\right)
\end{aligned}
$$

where $\lim _{k \rightarrow+\infty} o(1)=0$. Letting $k \rightarrow+\infty$, and then $A \rightarrow+\infty$, we get that

$$
\lim _{k \rightarrow+\infty} \int_{\Omega} \frac{\left|u_{k}\right|^{2^{\star}-\epsilon}}{|x|^{s}} d x=\int_{\Omega} \frac{\left|\tilde{u}_{\epsilon}\right|^{2^{\star}-\epsilon}}{|x|^{s}} d x
$$

It then follows that $\int_{\Omega} \frac{\left|\tilde{u}_{\epsilon}\right|^{2^{\star}-\epsilon}}{|x|^{s}} d x=1$. With the definition of $\mu_{s, a_{\epsilon}}^{\epsilon}(\Omega)$, we then get that

$$
\mu_{s, a_{\epsilon}}^{\epsilon}(\Omega) \leq \int_{\Omega}\left(\left|\nabla \tilde{u}_{\epsilon}\right|^{2}+a_{\epsilon} \tilde{u}_{\epsilon}^{2}\right) d x
$$

With (17), we then get that $\lim _{k \rightarrow+\infty} \theta_{k}=0$ in $H_{1,0}^{2}(\Omega)$. As a consequence, $\mu_{s, a_{\epsilon}}^{\epsilon}(\Omega)$ is attained by $\tilde{u}_{\epsilon}$. This proves the claim.

Up to replacing $\tilde{u}_{\epsilon}$ by $\left|\tilde{u}_{\epsilon}\right|$, we can assume that $\tilde{u}_{\epsilon} \geq 0$. We let

$$
u_{\epsilon}=\mu_{s, a_{\epsilon}}^{\epsilon}(\Omega)^{\frac{1}{2^{\star}-2-\epsilon}} \tilde{u}_{\epsilon} .
$$

As easily checked, $u_{\epsilon} \geq 0$ is also a minimizer for $\mu_{s, a_{\epsilon}}^{\epsilon}(\Omega)$. It satisfies

$$
\Delta u_{\epsilon}+a_{\epsilon} u_{\epsilon}=\frac{u_{\epsilon}^{2^{\star}-1-\epsilon}}{|x|^{s}} \text { in } \mathcal{D}^{\prime}(\Omega) .
$$

Moreover, it follows from the appendix and standard elliptic theory that $u_{\epsilon} \in$ $C^{1}(\bar{\Omega}) \cap C^{2}(\bar{\Omega} \backslash\{0\})$. Since $\Delta u_{\epsilon} \geq 0$ in $\Omega$ and $u_{\epsilon} \not \equiv 0$, it follows from the strong comparison principle that $u_{\epsilon}>0$ in $\Omega$.

Step 4.2: For any $\epsilon \in\left(0,2^{\star}-2\right)$, we let $\left(a_{\epsilon}\right), a$ as in (14) and (15). We let $\mu_{s, a_{\epsilon}}^{\epsilon}(\Omega)$ as in Proposition 4.1 We claim that

$$
\lim _{\epsilon \rightarrow 0} \mu_{s, a_{\epsilon}}^{\epsilon}(\Omega)=\mu_{s, a}(\Omega) .
$$

Indeed, we let $\alpha>0$ and let $u \in C_{c}^{\infty}(\Omega) \backslash\{0\}$ such that

$$
\frac{\int_{\Omega}\left(|\nabla u|^{2}+a u^{2}\right) d x}{\left(\int_{\Omega} \frac{|u|^{2^{\star}}}{|x|^{s}} d x\right)^{\frac{2}{2^{\star}}}} \leq \mu_{s, a}(\Omega)+\alpha .
$$


We have that

$$
\lim _{\epsilon \rightarrow 0} \frac{\int_{\Omega}\left(|\nabla u|^{2}+a_{\epsilon} u^{2}\right) d x}{\left(\int_{\Omega} \frac{|u|^{2^{\star}-\epsilon}}{|x|^{s}} d x\right)^{\frac{2}{2^{\star}-\epsilon}}}=\frac{\int_{\Omega}\left(|\nabla u|^{2}+a u^{2}\right) d x}{\left(\int_{\Omega} \frac{|u|^{2^{\star}}}{|x|^{s}} d x\right)^{\frac{2}{2^{\star}}}} \leq \mu_{s, a}(\Omega)+\alpha .
$$

Letting $\epsilon \rightarrow 0$ and $\alpha \rightarrow 0$, we get that

$$
\limsup _{\epsilon \rightarrow 0} \mu_{s, a_{\epsilon}}^{\epsilon}(\Omega) \leq \mu_{s, a}(\Omega) .
$$

We now let $v \in C_{c}^{\infty}(\Omega) \backslash\{0\}$. It follows from Hölder's inequality that

$$
\left(\int_{\Omega} \frac{|v|^{2^{\star}-\epsilon}}{|x|^{s}} d x\right)^{\frac{2}{2^{\star}-\epsilon}} \leq\left(\int_{\Omega} \frac{d x}{|x|^{s}}\right)^{\frac{2 \epsilon}{2^{\star} \cdot\left(2^{\star}-\epsilon\right)}}\left(\int_{\Omega} \frac{|v|^{2^{\star}}}{|x|^{s}} d x\right)^{\frac{2}{2^{\star}}}
$$

and then

$$
\begin{aligned}
\frac{\int_{\Omega}\left(|\nabla v|^{2}+a v^{2}\right) d x}{\left(\int_{\Omega} \frac{|v|^{\star}}{|x|^{s}} d x\right)^{\frac{2}{2^{\star}}} \leq} & \left(\int_{\Omega} \frac{d x}{|x|^{s}}\right)^{\frac{2 \epsilon}{2^{\star} \cdot\left(2^{\star}-\epsilon\right)}} \cdot \frac{\int_{\Omega}\left(|\nabla v|^{2}+a_{\epsilon} v^{2}\right) d x}{\left(\int_{\Omega} \frac{|v|^{2^{\star}}-\epsilon}{|x|^{s}} d x\right)^{\frac{2}{2^{\star}-\epsilon}}} \\
& +\frac{\int_{\Omega}\left(a-a_{\epsilon}\right) v^{2} d x}{\left(\int_{\Omega} \frac{|v|^{2^{\star}-\epsilon}}{|x|^{s}} d x\right)^{\frac{2}{2^{\star}-\epsilon}}}
\end{aligned}
$$

for $\epsilon>0$ small. Here, we have used that $\Delta+a_{\epsilon}$ is coercive on $\Omega$ for $\epsilon>0$ small, which is a consequence of (14) and (15). Taking the infimum, using Hölder's inequality and (14), we get that

$$
\mu_{s, a}(\Omega) \leq(1+o(1)) \mu_{s, a_{\epsilon}}^{\epsilon}(\Omega)
$$

where $\lim _{\epsilon \rightarrow 0} o(1)=0$. The conclusion of Step 4.2 then follows from (18) and (19).

Step 4.3: We prove that, when it is nonzero, the weak limit of the $u_{\epsilon}$ 's is a minimizer for $\mu_{s, a}(\Omega)$. This is the object of the following proposition.

Proposition 4.2. Let $\Omega$ be a smooth bounded domain of $\mathbb{R}^{n}, n \geq 3$, such that $0 \in \partial \Omega$. For $s \in(0,2)$ and $\epsilon \in\left(0,2^{\star}-2\right)$, we let $a_{\epsilon}, a$ be as in (14) and (15). For any $\epsilon \in\left(0,2^{\star}-2\right)$, let $\mu_{s, a_{\epsilon}}^{\epsilon}(\Omega)$ and $u_{\epsilon}$ be as in Proposition 4.1. Then there exists $u_{0} \in H_{1,0}^{2}(\Omega)$ such that, up to a subsequence, $u_{\epsilon} \rightarrow u_{0}$ weakly in $H_{1,0}^{2}(\Omega)$ when $\epsilon \rightarrow 0$. If $u_{0} \not \equiv 0$, then $\lim _{\epsilon \rightarrow 0} u_{\epsilon}=u_{0}$ strongly in $H_{1,0}^{2}(\Omega)$ and $u_{0}$ is a minimizer for $\mu_{s, a}(\Omega)$. In particular, $\mu_{s, a}(\Omega)$ is attained.

Proof. It is clear from Proposition 4.1 and the hypothesis (14) and (15) that

$$
\left\|u_{\epsilon}\right\|_{H_{1,0}^{2}(\Omega)}=O(1)
$$

when $\epsilon \rightarrow 0$. Then there exists $u_{0} \in H_{1,0}^{2}(\Omega)$ such that, up to a subsequence, $u_{\epsilon} \rightarrow u_{0}$ weakly in $H_{1,0}^{2}(\Omega)$ when $\epsilon \rightarrow 0$. We assume that $u_{0} \not \equiv 0$. It then follows from the definition of $\mu_{s, a}(\Omega)$ that

$$
\frac{\int_{\Omega}\left(\left|\nabla u_{0}\right|^{2}+a u_{0}^{2}\right) d x}{\left(\int_{\Omega} \frac{\left|u_{0}\right|^{\star}}{|x|^{s}} d x\right)^{\frac{2}{2^{\star}}}} \geq \mu_{s, a}(\Omega) .
$$

Testing the weak inequality $\Delta u_{\epsilon}+a_{\epsilon} u_{\epsilon}=\frac{u_{\epsilon}^{2^{\star}-1-\epsilon}}{|x|^{s}}$ on $u_{0}$ and letting $\epsilon \rightarrow 0$, we get that

$$
\int_{\Omega}\left(\left|\nabla u_{0}\right|^{2}+a u_{0}^{2}\right) d x=\int_{\Omega} \frac{\left|u_{0}\right|^{2^{\star}}}{|x|^{s}} d x .
$$


We then obtain that

$$
\int_{\Omega} \frac{\left|u_{0}\right|^{2^{\star}}}{|x|^{s}} d x \geq \mu_{s, a}(\Omega)^{\frac{2^{\star}}{2^{\star}-2}}
$$

Since $u_{\epsilon} \rightarrow u_{0}$ when $\epsilon \rightarrow 0$, we get with the definition of $u_{\epsilon}$ in Proposition 4.1 and Step 4.2 that

$$
\int_{\Omega} \frac{\left|u_{0}\right|^{2^{\star}}}{|x|^{s}} d x \leq \liminf _{\epsilon \rightarrow 0} \int_{\Omega} \frac{\left|u_{\epsilon}\right|^{2^{\star}-\epsilon}}{|x|^{s}} d x=\mu_{s, a}(\Omega)^{\frac{2^{\star}}{2^{\star}-2}} .
$$

Consequently, we get that

$$
\int_{\Omega}\left(\left|\nabla u_{0}\right|^{2}+a u_{0}^{2}\right) d x=\int_{\Omega} \frac{\left|u_{0}\right|^{2^{\star}}}{|x|^{s}} d x=\mu_{s, a}(\Omega)^{\frac{2^{\star}}{2^{\star}-2}} .
$$

Since $\mu_{s, a_{\epsilon}}^{\epsilon}(\Omega)^{\frac{2^{\star}-\epsilon}{2^{\star}-2-\epsilon}}=\int_{\Omega}\left(\left|\nabla u_{\epsilon}\right|^{2}+a_{\epsilon} u_{\epsilon}^{2}\right) d x$, we get with the definition of $u_{\epsilon}$ in Proposition 4.1 that

$$
\mu_{s, a}(\Omega)^{\frac{2^{\star}}{2^{\star}-2}}=\int_{\Omega}\left(\left|\nabla u_{0}\right|^{2}+a u_{0}^{2}\right) d x+\int_{\Omega}\left|\nabla\left(u_{\epsilon}-u_{0}\right)\right|^{2} d x+o(1)
$$

with $\lim _{\epsilon \rightarrow 0} o(1)=0$. It follows from (20) and (21) that $\lim _{\epsilon \rightarrow 0} u_{\epsilon}=u_{0}$ in $H_{1,0}^{2}(\Omega)$. As easily checked, in this case, $u_{0}$ is a minimizer for $\mu_{s, a}(\Omega)$.

\section{Preliminary Blow-Up analysis}

From now on, we let $\Omega$ be a smooth bounded domain of $\mathbb{R}^{n}, n \geq 3$, such that $0 \in \partial \Omega$. We let $s \in(0,2)$. For any $\epsilon>0$, we let $p_{\epsilon} \in\left[0,2^{\star}-2\right)$ such that

$$
\lim _{\epsilon \rightarrow 0} p_{\epsilon}=0 .
$$

We let $a \in C^{1}(\bar{\Omega})$ and a family $\left(a_{\epsilon}\right)_{\epsilon>0} \in C^{1}(\bar{\Omega})$ such that (14) and (15) hold. For any $\epsilon>0$, we consider $u_{\epsilon} \in H_{1,0}^{2}(\Omega) \cap C^{2}(\bar{\Omega} \backslash\{0\})$ a solution to the system

$$
\begin{cases}\Delta u_{\epsilon}+a_{\epsilon} u_{\epsilon}=\frac{u_{\epsilon}^{2^{\star}-1-p_{\epsilon}}}{|x|^{s}} & \text { in } \mathcal{D}^{\prime}(\Omega) \\ u_{\epsilon}>0 & \text { in } \Omega\end{cases}
$$

for all $\epsilon>0$. We assume that $u_{\epsilon}$ is of minimal energy type, that is

$$
\int_{\Omega} \frac{\left|u_{\epsilon}\right|^{2^{\star}-p_{\epsilon}}}{|x|^{s}} d x=\mu_{s}(\Omega)^{\frac{2^{\star}}{2^{\star}-2}}+o(1)
$$

where $\lim _{\epsilon \rightarrow 0} o(1)=0$. Note that it follows from (14), (15), (23) and (24) that

$$
\left\|u_{\epsilon}\right\|_{H_{1,0}^{2}(\Omega)}=O(1)
$$

when $\epsilon \rightarrow 0$. We also assume that blow-up occurs, that is

$$
u_{\epsilon} \rightarrow 0
$$

weakly in $H_{1,0}^{2}(\Omega)$ when $\epsilon \rightarrow 0$. Such a family arises naturally when $u_{0} \equiv 0$ in Propositions 4.1 and 4.2 In the remaining sections, we describe precisely the behaviour of the $u_{\epsilon}$ 's. We follow the strategy developed in [10].

It follows from Proposition 8.1 of the Appendix that $u_{\epsilon} \in C^{0}(\bar{\Omega})$. We let $x_{\epsilon} \in \Omega$ and $\mu_{\epsilon}, k_{\epsilon}>0$ such that

$$
\max _{\Omega} u_{\epsilon}=u_{\epsilon}\left(x_{\epsilon}\right)=\mu_{\epsilon}^{-\frac{n-2}{2}} \text { and } k_{\epsilon}:=\mu_{\epsilon}^{1-\frac{p_{\epsilon}}{2^{\star}-2}} .
$$


We let $\varphi: U \rightarrow V$ a local chart as in (16) with $x_{0}=0$, where $U, V$ are open neighborhoods of 0 . For any $\epsilon>0$ and any $x \in \frac{U}{k_{\epsilon}} \cap\left\{x_{1} \leq 0\right\}$, we define the maximum rescaling of $u_{\epsilon}$ as follows

$$
v_{\epsilon}(x):=\frac{u_{\epsilon} \circ \varphi\left(k_{\epsilon} x\right)}{u_{\epsilon}\left(x_{\epsilon}\right)},
$$

where $x_{\epsilon}, k_{\epsilon}$ are as in (27). As easily checked, for any $\eta \in C_{c}^{\infty}\left(\mathbb{R}^{n}\right)$, we have that $\eta v_{\epsilon} \in H_{1,0}^{2}\left(\mathbb{R}_{-}^{n}\right)$. In this section, we prove the following proposition:

Proposition 5.1. Let $\Omega$ be a smooth bounded domain of $\mathbb{R}^{n}, n \geq 3$ and $s \in(0,2)$. Consider $\left(p_{\epsilon}\right)_{\epsilon>0}$ such that $p_{\epsilon} \in\left[0,2^{\star}-2\right)$ for all $\epsilon>0$. We consider $\left(u_{\epsilon}\right)_{\epsilon>0} \in$ $H_{1,0}^{2}(\Omega)$ such that (14), (15), (23), (24) and (26) hold. Let $v_{\epsilon}$ be as in (28). Then there exists $v \in H_{1,0}^{2}\left(\mathbb{R}_{-}^{n}\right) \backslash\{0\}$ such that for any $\eta \in C_{c}^{\infty}\left(\mathbb{R}^{n}\right)$,

$$
\eta v_{\epsilon} \rightarrow \eta v \text { in } H_{1,0}^{2}\left(\mathbb{R}_{-}^{n}\right)
$$

when $\epsilon \rightarrow 0$. Moreover, $v$ verifies that

$$
\Delta v=\frac{v^{2^{\star}-1}}{|x|^{s}} \text { in } \mathcal{D}^{\prime}\left(\mathbb{R}_{-}^{n}\right)
$$

and

$$
\int_{\mathbb{R}_{-}^{n}}|\nabla v|^{2} d x=\mu_{s}(\Omega)^{\frac{2^{\star}}{2^{\star}-2}}=\mu_{s}\left(\mathbb{R}_{-}^{n}\right)^{\frac{2^{\star}}{2{ }^{\star}-2}} .
$$

In addition, there exists $\theta \in(0,1)$ such that $v \in C^{1, \theta}\left(\overline{\mathbb{R}_{-}^{n}}\right)$ and

$$
v_{\epsilon} \rightarrow v \text { in } C_{l o c}^{1, \theta}\left(\overline{\mathbb{R}_{-}^{n}}\right)
$$

when $\epsilon \rightarrow 0$. Moreover, we have that

$$
\lim _{\epsilon \rightarrow 0} \mu_{\epsilon}^{p_{\epsilon}}=1 .
$$

Proof. Steps 5.1 to 5.9 below are devoted to the proof of this Proposition.

Step 5.1: We claim that

$$
\mu_{\epsilon}=o(1)
$$

when $\epsilon \rightarrow 0$. We proceed by contradiction and assume that $\lim _{\epsilon \rightarrow 0} \mu_{\epsilon} \neq 0$. In this case, up to a subsequence, there exists $C>0$ such that $u_{\epsilon}(x) \leq C$ for all $x \in \Omega$ and all $\epsilon>0$. Since (26) hold, it follows from standard elliptic theory (see for instance [20]) that $\lim _{\epsilon \rightarrow 0} u_{\epsilon}=0$ in $C^{0}(\bar{\Omega})$. A contradiction with (24). This proves (30).

Step 5.2: We claim that

$$
\left|x_{\epsilon}\right|=O\left(k_{\epsilon}\right)
$$

when $\epsilon \rightarrow 0$. We proceed by contradiction and assume that

$$
\lim _{\epsilon \rightarrow 0} \frac{\left|x_{\epsilon}\right|}{k_{\epsilon}}=+\infty .
$$

For any $\epsilon>0$, we let

$$
\beta_{\epsilon}=\left|x_{\epsilon}\right|^{\frac{s}{2}} u_{\epsilon}\left(x_{\epsilon}\right)^{\frac{2+p_{\epsilon}-2^{\star}}{2}}=\left|x_{\epsilon}\right|^{\frac{s}{2}} k_{\epsilon}^{\frac{2-s}{2}} .
$$

It follows from the definition (33) of $\beta_{\epsilon}$ and (32) that

$$
\lim _{\epsilon \rightarrow 0} \beta_{\epsilon}=0, \lim _{\epsilon \rightarrow 0} \frac{\beta_{\epsilon}}{k_{\epsilon}}=+\infty \text { and } \lim _{\epsilon \rightarrow 0} \frac{\beta_{\epsilon}}{\left|x_{\epsilon}\right|}=0
$$

when $\epsilon \rightarrow 0$. 
Case 5.2.1: We assume that there exists $\rho>0$ such that

$$
\frac{d\left(x_{\epsilon}, \partial \Omega\right)}{\beta_{\epsilon}} \geq 2 \rho
$$

for all $\epsilon>0$. For $x \in B_{2 \rho}(0)$ and $\epsilon>0$, we define

$$
\bar{v}_{\epsilon}(x):=\frac{u_{\epsilon}\left(x_{\epsilon}+\beta_{\epsilon} x\right)}{u_{\epsilon}\left(x_{\epsilon}\right)} .
$$

Note that this is well defined since $x_{\epsilon}+\beta_{\epsilon} x \in \Omega$ for all $x \in B_{2 \rho}(0)$. As easily checked, we have that

$$
\Delta \bar{v}_{\epsilon}+\beta_{\epsilon}^{2} a_{\epsilon}\left(x_{\epsilon}+\beta_{\epsilon} x\right) \bar{v}_{\epsilon}=\frac{\bar{v}_{\epsilon}^{2^{\star}-1-p_{\epsilon}}}{\left|\frac{x_{\epsilon}}{\left|x_{\epsilon}\right|}+\frac{\beta_{\epsilon}}{\left|x_{\epsilon}\right|} \cdot x\right|^{s}}
$$

weakly in $B_{2 \rho}(0)$. Since (34) holds, we have that

$$
\Delta \bar{v}_{\epsilon}+\beta_{\epsilon}^{2} a_{\epsilon}\left(x_{\epsilon}+\beta_{\epsilon} x\right) \bar{v}_{\epsilon}=(1+o(1)) \bar{v}_{\epsilon}^{2^{\star}-1-p_{\epsilon}}
$$

weakly in $B_{2 \rho}(0)$, where $\lim _{\epsilon \rightarrow 0} o(1)=0$ in $C_{l o c}^{0}\left(B_{2 \rho}(0)\right)$. Since $0 \leq \bar{v}_{\epsilon}(x) \leq$ $\bar{v}_{\epsilon}(0)=1$ for all $x \in B_{2 \rho}(0)$, it follows from standard elliptic theory that there exists $v \in C^{1}\left(B_{2 \rho}(0)\right)$ such that $v \geq 0$ and

$$
\bar{v}_{\epsilon} \rightarrow \bar{v}
$$

in $C_{l o c}^{1}\left(B_{2 \rho}(0)\right)$ when $\epsilon \rightarrow 0$. In particular,

$$
\bar{v}(0)=\lim _{\epsilon \rightarrow 0} \bar{v}_{\epsilon}(0)=1 .
$$

With a change of variables and the definition (33) of $\beta_{\epsilon}$, we get that

$$
\begin{aligned}
& \int_{\Omega \cap B_{\rho \beta_{\epsilon}}\left(x_{\epsilon}\right)} \frac{u_{\epsilon}^{2^{\star}-p_{\epsilon}}}{|x|^{s}} d x=\frac{u_{\epsilon}\left(x_{\epsilon}\right)^{2^{\star}-p_{\epsilon}} \beta_{\epsilon}^{n}}{\left|x_{\epsilon}\right|^{s}} \int_{B_{\rho}(0)} \frac{\bar{v}_{\epsilon}^{2^{\star}-p_{\epsilon}}}{\left|\frac{x_{\epsilon}}{\mid x_{\epsilon}}+\frac{\beta_{\epsilon}}{\left|x_{\epsilon}\right|} \cdot x\right|^{s}} d x \\
& \geq\left(\frac{\beta_{\epsilon}}{k_{\epsilon}}\right)^{n-2} \int_{B_{\rho}(0)} \frac{\bar{v}_{\epsilon}^{2^{\star}-p_{\epsilon}}}{\left|\frac{x_{\epsilon}}{\left|x_{\epsilon}\right|}+\frac{\beta_{\epsilon}}{\left|x_{\epsilon}\right|} x\right|^{s}} d x .
\end{aligned}
$$

Using (24), (34) and passing to the limit $\epsilon \rightarrow 0$ (note that $\mu_{\epsilon}^{-1} \geq 1$ for $\epsilon>0$ small), we get that

$$
\int_{B_{\rho}(0)} \bar{v}^{2^{\star}} d x=0
$$

and then $\bar{v} \equiv 0$ in $B_{\rho}(0)$. A contradiction with (35). Then (32) does not hold. This proves that (31) holds in Case 5.2.1.

Case 5.2.2: We assume that, up to a subsequence,

$$
\lim _{\epsilon \rightarrow 0} \frac{d\left(x_{\epsilon}, \partial \Omega\right)}{\beta_{\epsilon}}=0
$$

In this case,

$$
\lim _{\epsilon \rightarrow 0} x_{\epsilon}=x_{0} \in \partial \Omega
$$

Since $x_{0} \in \partial \Omega$, we let $\varphi: U \rightarrow V$ as in (16), where $U, V$ are open neighborhoods of 0 and $x_{0}$ respectively. We let $\tilde{u}_{\epsilon}=u_{\epsilon} \circ \varphi$, which is defined on $U \cap\left\{x_{1} \leq 0\right\}$. For any $i, j=1, \ldots, n$, we let $g_{i j}=\left(\partial_{i} \varphi, \partial_{j} \varphi\right)$, where $(\cdot, \cdot)$ denotes the Euclidean scalar 
product on $\mathbb{R}^{n}$, and we consider $g$ as a metric on $\mathbb{R}^{n}$. We let $\Delta_{g}=-\operatorname{div}_{g}(\nabla)$ the Laplace-Beltrami operator with respect to the metric $g$. In our basis, we have that

$$
\Delta_{g}=-g^{i j}\left(\partial_{i j}-\Gamma_{i j}^{k} \partial_{k}\right),
$$

where $g^{i j}=\left(g^{-1}\right)_{i j}$ are the coordinates of the inverse of the tensor $g$ and the $\Gamma_{i j}^{k}$ 's are the Christoffel symbols of the metric $g$. As easily checked, we have that

$$
\Delta_{g} \tilde{u}_{\epsilon}+a_{\epsilon} \circ \varphi(x) \cdot \tilde{u}_{\epsilon}=\frac{\tilde{u}_{\epsilon}^{2^{\star}-1-p_{\epsilon}}}{|\varphi(x)|^{s}}
$$

weakly in $U \cap\left\{x_{1}<0\right\}$. We let $z_{\epsilon} \in \partial \Omega$ such that

$$
\left|z_{\epsilon}-x_{\epsilon}\right|=d\left(x_{\epsilon}, \partial \Omega\right) \text {. }
$$

We let $\tilde{x}_{\epsilon}, \tilde{z}_{\epsilon} \in U$ such that

$$
\varphi\left(\tilde{x}_{\epsilon}\right)=x_{\epsilon} \text { and } \varphi\left(\tilde{z}_{\epsilon}\right)=z_{\epsilon} .
$$

It follows from the properties (16) of $\varphi$ that

$$
\lim _{\epsilon \rightarrow 0} \tilde{x}_{\epsilon}=\lim _{\epsilon \rightarrow 0} \tilde{z}_{\epsilon}=0,\left(\tilde{x}_{\epsilon}\right)_{1}<0 \text { and }\left(\tilde{z}_{\epsilon}\right)_{1}=0 .
$$

At last, we let

$$
\tilde{v}_{\epsilon}(x):=\frac{\tilde{u}_{\epsilon}\left(\tilde{z}_{\epsilon}+\beta_{\epsilon} x\right)}{\tilde{u}_{\epsilon}\left(\tilde{x}_{\epsilon}\right)}
$$

for all $x \in \frac{U-\tilde{z}_{\epsilon}}{\beta_{\epsilon}} \cap\left\{x_{1}<0\right\}$. With (39), we get that $\tilde{v}_{\epsilon}$ is defined on $B_{R}(0) \cap\left\{x_{1}<0\right\}$ for all $R>0$, as soon as $\epsilon$ is small enough. The function $\tilde{v}_{\epsilon}$ verifies

$$
\Delta_{\tilde{g}_{\epsilon}} \tilde{v}_{\epsilon}+\beta_{\epsilon}^{2} a_{\epsilon} \circ \varphi\left(\tilde{z}_{\epsilon}+\beta_{\epsilon} x\right) \tilde{v}_{\epsilon}=\frac{\tilde{v}_{\epsilon}^{2^{\star}-1-p_{\epsilon}}}{\left|\frac{\varphi\left(\tilde{z}_{\epsilon}+\beta_{\epsilon} x\right)}{\left|x_{\epsilon}\right|}\right|^{s}}
$$

weakly in $B_{R}(0) \cap\left\{x_{1}<0\right\}$. In this expression, $\tilde{g}_{\epsilon}=g\left(\tilde{z}_{\epsilon}+\beta_{\epsilon} x\right)$ and $\Delta_{\tilde{g}_{\epsilon}}$ is the Laplace-Beltrami operator with respect to the metric $\tilde{g}_{\epsilon}$. With (36), (37) and (38), we get that

$$
\varphi\left(\tilde{z}_{\epsilon}+\beta_{\epsilon} x\right)=x_{\epsilon}+O_{R}(1) \beta_{\epsilon},
$$

for all $x \in B_{R}(0) \cap\left\{x_{1} \leq 0\right\}$ and all $\epsilon>0$, where there exists $C_{R}>0$ such that $\left|O_{R}(1)\right| \leq C_{R}$ for all $x \in B_{R}(0) \cap\left\{x_{1}<0\right\}$. With (34), we then get that

$$
\lim _{\epsilon \rightarrow 0} \frac{\left|\varphi\left(\tilde{z}_{\epsilon}+\beta_{\epsilon} x\right)\right|}{\left|x_{\epsilon}\right|}=1
$$

in $C^{0}\left(B_{R}(0) \cap\left\{x_{1} \leq 0\right\}\right)$. It then follows that

$$
\Delta_{\tilde{g}_{\epsilon}} \tilde{v}_{\epsilon}+\beta_{\epsilon}^{2} a_{\epsilon} \circ \varphi\left(\tilde{z}_{\epsilon}+\beta_{\epsilon} x\right) \tilde{v}_{\epsilon}=(1+o(1)) \tilde{v}_{\epsilon}^{2^{\star}-1-p_{\epsilon}}
$$

weakly in $B_{R}(0) \cap\left\{x_{1}<0\right\}$, where $\lim _{\epsilon \rightarrow 0} o(1)=0$ in $C^{0}\left(B_{R}(0) \cap\left\{x_{1} \leq 0\right\}\right)$. Since $\tilde{v}_{\epsilon}$ vanishes on $B_{R}(0) \cap\left\{x_{1}=0\right\}$ (in the sense of the trace) and that $0 \leq \tilde{v}_{\epsilon} \leq 1$, it follows from standard elliptic theory that there exists $\tilde{v} \in C^{1}\left(B_{R}(0) \cap\left\{x_{1} \leq 0\right\}\right)$ such that

$$
\lim _{\epsilon \rightarrow 0} \tilde{v}_{\epsilon}=\tilde{v}
$$

in $C^{0}\left(B_{\frac{R}{2}}(0) \cap\left\{x_{1} \leq 0\right\}\right)$. In particular,

$$
\tilde{v} \equiv 0 \text { on } B_{\frac{R}{2}}(0) \cap\left\{x_{1}=0\right\} .
$$


Moreover, it follows from (37) and (38) that

$$
\tilde{v}_{\epsilon}\left(\frac{\tilde{x}_{\epsilon}-\tilde{z}_{\epsilon}}{\beta_{\epsilon}}\right)=1 \text { and } \lim _{\epsilon \rightarrow 0} \frac{\tilde{x}_{\epsilon}-\tilde{z}_{\epsilon}}{\beta_{\epsilon}}=0 .
$$

In particular, $\tilde{v}(0)=1$. A contradiction with (40). Then (32) does not hold. This proves (31) in Case 5.2.2.

In both cases, we have obtained that (31) holds. This concludes Step 5.2.

A consequence of (31) is that $\lim _{\epsilon \rightarrow 0} x_{\epsilon}=0 \in \partial \Omega$. We let $\varphi: U \rightarrow V$ as in (16) be a local chart of $\partial \Omega$ with $x_{0}=0$ (in other words, $\varphi(0)=0$ ), where $U, V$ are open neighborhoods of 0 . We write

$$
x_{\epsilon}=\varphi\left(x_{1, \epsilon}, z_{\epsilon}\right),
$$

where $x_{1, \epsilon}<0$ and $z_{\epsilon} \in \mathbb{R}^{n-1}$ are such that $\left(x_{1, \epsilon}, z_{\epsilon}\right) \in U$.

Step 5.3: We claim that

$$
d\left(x_{\epsilon}, \partial \Omega\right)=(1+o(1))\left|x_{1, \epsilon}\right|=O\left(k_{\epsilon}\right) \text { and } z_{\epsilon}=O\left(k_{\epsilon}\right),
$$

when $\epsilon \rightarrow 0$. Indeed, with (31), we get that

$$
d\left(x_{\epsilon}, \partial \Omega\right) \leq\left|x_{\epsilon}\right|=O\left(k_{\epsilon}\right)
$$

when $\epsilon \rightarrow 0$. We first remark that

$$
d\left(x_{\epsilon}, \partial \Omega\right) \leq d\left(x_{\epsilon}, \varphi\left(0, z_{\epsilon}\right)\right)=\left|x_{1, \epsilon}\right| .
$$

We let $a_{\epsilon} \in \operatorname{span}\left(\vec{e}_{2}, \ldots, \vec{e}_{n}\right)$ and $Y_{\epsilon}=\varphi\left(0, a_{\epsilon}\right) \in \partial \Omega$ such that $d\left(x_{\epsilon}, \partial \Omega\right)=\left|x_{\epsilon}-Y_{\epsilon}\right|$. Since $d\left(x_{\epsilon}, \partial \Omega\right) \leq\left|x_{1, \epsilon}\right|$, we get that

$$
z_{\epsilon}-a_{\epsilon}=O\left(\left|x_{1, \epsilon}\right|\right)
$$

when $\epsilon \rightarrow 0$. Since $\nabla \varphi_{0}(0)=0$ (where $\varphi_{0}$ is as in (16)), we get that

$$
\varphi_{0}\left(z_{\epsilon}\right)=\varphi_{0}\left(a_{\epsilon}\right)+o\left(\left|z_{\epsilon}-a_{\epsilon}\right|\right)=\varphi_{0}\left(a_{\epsilon}\right)+o\left(\left|x_{1, \epsilon}\right|\right)
$$

when $\epsilon \rightarrow 0$. Moreover,

$$
\begin{aligned}
d\left(x_{\epsilon}, \partial \Omega\right) & =\left|x_{\epsilon}-Y_{\epsilon}\right| \\
& =\left|\left(x_{1, \epsilon}+\varphi_{0}\left(z_{\epsilon}\right)-\varphi_{0}\left(a_{\epsilon}\right), z_{\epsilon}-a_{\epsilon}\right)\right| \\
& =\left|\left(x_{1, \epsilon}+o\left(\left|x_{1, \epsilon}\right|\right), z_{\epsilon}-a_{\epsilon}\right)\right| \leq\left|x_{1, \epsilon}\right|
\end{aligned}
$$

when $\epsilon \rightarrow 0$. It then follows that $z_{\epsilon}-a_{\epsilon}=o\left(\left|x_{1, \epsilon}\right|\right)$ and $d\left(x_{\epsilon}, \partial \Omega\right)=(1+o(1))\left|x_{1, \epsilon}\right|$ when $\epsilon \rightarrow 0$. This last result, (31) and (42) prove (41).

Step 5.4: We let

$$
\lambda_{\epsilon}:=-\frac{x_{1, \epsilon}}{k_{\epsilon}}>0 \text { and } \theta_{\epsilon}:=\frac{z_{\epsilon}}{k_{\epsilon}} .
$$

It follows from (41) that there exist $\lambda_{0} \geq 0$ and $\theta_{0} \in \mathbb{R}^{n-1}$ such that

$$
\lim _{\epsilon \rightarrow 0} \lambda_{\epsilon}=\lambda_{0} \text { and } \lim _{\epsilon \rightarrow 0} \theta_{\epsilon}=\theta_{0} .
$$

For any $\epsilon>0$ and any $x \in \frac{U}{k_{\epsilon}} \cap\left\{x_{1} \leq 0\right\}$, we let (as in (28))

$$
v_{\epsilon}(x):=\frac{u_{\epsilon} \circ \varphi\left(k_{\epsilon} x\right)}{u_{\epsilon}\left(x_{\epsilon}\right)},
$$

where $\varphi: U \rightarrow V$ is defined in (16) (with $x_{0}=0$ ) and $k_{\epsilon}, x_{\epsilon}$ are as in (27). As easily checked, for any $\eta \in C_{c}^{\infty}\left(\mathbb{R}^{n}\right)$, we have that

$$
\eta v_{\epsilon} \in H_{1,0}^{2}\left(\mathbb{R}_{-}^{n}\right)
$$


for all $\epsilon>0$. We go on in the proof of Proposition 5.1

Step 5.5: We claim that for any $\eta \in C_{c}^{\infty}\left(\mathbb{R}^{n}\right)$, there exists $v_{\eta} \in H_{1,0}^{2}\left(\mathbb{R}_{-}^{n}\right)$ such that, up to a subsequence,

$$
\eta v_{\epsilon} \rightarrow v_{\eta}
$$

weakly in $H_{1,0}^{2}\left(\mathbb{R}_{-}^{n}\right)$. Indeed, as easily checked, we have that

$$
\nabla\left(\eta v_{\epsilon}\right)(x)=v_{\epsilon} \nabla \eta+\frac{k_{\epsilon}}{u_{\epsilon}\left(x_{\epsilon}\right)} \eta \cdot D_{\left(k_{\epsilon} x\right)} \varphi\left[\left(\nabla u_{\epsilon}\right)\left(\varphi\left(k_{\epsilon} x\right)\right)\right],
$$

for all $\epsilon>0$ and all $x \in \mathbb{R}_{-}^{n}$. In this expression, $D_{x} \varphi$ is the differential of the function $\varphi$ at $x$. It is standard that for any $\alpha>0$, there exists $C_{\alpha}>0$ such that

$$
(x+y)^{2} \leq C_{\alpha} x^{2}+(1+\alpha) \cdot y^{2}
$$

for all $x, y>0$. With this inequality, we get that

$$
\begin{aligned}
& \int_{\mathbb{R}_{-}^{n}}\left|\nabla\left(\eta v_{\epsilon}\right)\right|^{2} d x \leq C_{\alpha} \int_{\mathbb{R}_{-}^{n}}|\nabla \eta|^{2} v_{\epsilon}^{2} d x \\
& +(1+\alpha) \int_{\mathbb{R}_{-}^{n}} \eta^{2} \frac{k_{\epsilon}^{2}}{u_{\epsilon}\left(x_{\epsilon}\right)^{2}} \cdot\left|D_{\left(k_{\epsilon} x\right)} \varphi\left[\left(\nabla u_{\epsilon}\right)\left(\varphi\left(k_{\epsilon} x\right)\right)\right]\right|^{2} d x .
\end{aligned}
$$

Since $D_{0} \varphi=I d_{\mathbb{R}^{n}}$, we get that with Hölder's inequality and a change of variables that

$$
\begin{aligned}
& \int_{\mathbb{R}_{-}^{n}}\left|\nabla\left(\eta v_{\epsilon}\right)\right|^{2} d x \leq C_{\alpha} \int_{\mathbb{R}_{-}^{n}}|\nabla \eta|^{2} v_{\epsilon}^{2} d x \\
& +(1+\alpha) \cdot\left(1+O\left(k_{\epsilon}\right)\right) \int_{\mathbb{R}_{-}^{n}} \eta^{2} \frac{k_{\epsilon}^{2}}{u_{\epsilon}\left(x_{\epsilon}\right)^{2}} \cdot\left|\nabla u_{\epsilon}\right|^{2}\left(\varphi\left(k_{\epsilon} x\right)\right) d x \\
& \leq C_{\alpha}\|\nabla \eta\|_{n}^{2} \cdot\left\|v_{\epsilon}\right\|_{L^{\frac{2 n}{n-2}}}^{2}\left(\operatorname{Supp}_{p_{\epsilon}(n)} \int_{\Omega}\left|\nabla u_{\epsilon}\right|^{2} d x\right. \\
& +(1+\alpha) \cdot\left(1+O\left(k_{\epsilon}\right)\right) \cdot \mu_{\epsilon}^{\frac{\left.p^{2}-2\right)}{2^{x}-2}} \int_{\Omega}
\end{aligned}
$$

With another change of variables, we get that

$$
\begin{aligned}
& \int_{\mathbb{R}_{-}^{n}}\left|\nabla\left(\eta v_{\epsilon}\right)\right|^{2} d x \leq C_{\alpha} \cdot \mu_{\epsilon}^{\frac{(n-2) p_{\epsilon}}{2^{\star}-2}}\|\nabla \eta\|_{n}^{2} \cdot\left\|u_{\epsilon}\right\|_{L^{\frac{2 n}{n-2}}(\Omega)}^{2} \\
& +(1+\alpha) \cdot\left(1+O\left(k_{\epsilon}\right)\right) \cdot \mu_{\epsilon}^{\frac{p_{\epsilon}(n-2)}{2^{\star}-2}} \int_{\Omega}\left|\nabla u_{\epsilon}\right|^{2} d x .
\end{aligned}
$$

With (25), Sobolev's inequality and since $\mu_{\epsilon}^{p_{\epsilon}} \leq 1$ for all $\epsilon>0$ small enough, we get with (47) that

$$
\left\|\eta v_{\epsilon}\right\|_{H_{1,0}^{2}\left(\mathbb{R}_{-}^{n}\right)}=O(1)
$$

when $\epsilon \rightarrow 0$. It then follows that there exists $v_{\eta} \in H_{1,0}^{2}\left(\mathbb{R}_{-}^{n}\right)$ such that, up to a subsequence, $\eta v_{\epsilon} \rightarrow v_{\eta}$ weakly in $H_{1,0}^{2}\left(\mathbb{R}_{-}^{n}\right)$ when $\epsilon \rightarrow 0$. This concludes Step 5.5.

Step 5.6: We claim that there exists $v \in H_{1,0}^{2}\left(\mathbb{R}_{-}^{n}\right)$ such that for any $\eta \in C_{c}^{\infty}\left(\mathbb{R}^{n}\right)$, we have that, up to a subsequence,

$$
\eta v_{\epsilon} \rightarrow \eta v
$$

weakly in $H_{1,0}^{2}\left(\mathbb{R}_{-}^{n}\right)$ when $\epsilon \rightarrow 0$. Indeed, we let $\eta_{1} \in C_{c}^{\infty}\left(\mathbb{R}^{n}\right)$ such that $\eta_{1} \equiv 1$ in $B_{1}(0)$ and $\eta_{1} \equiv 0$ in $\mathbb{R}^{n} \backslash B_{2}(0)$. For any $R>0$, we let $\eta_{R}(x)=\eta_{1}\left(\frac{x}{R}\right)$ for all 
$x \in \mathbb{R}^{n}$. With a diagonal argument, we can assume that, up to a subsequence, for any $R>0$, there exists $v_{R} \in H_{1,0}^{2}\left(\mathbb{R}_{-}^{n}\right)$ such that

$$
\eta_{R} v_{\epsilon} \rightarrow v_{R}
$$

weakly in $H_{1,0}^{2}\left(\mathbb{R}_{-}^{n}\right)$ when $\epsilon \rightarrow 0$, and that $\left(\eta_{R} v_{\epsilon}\right)(x) \rightarrow v_{R}(x)$ when $\epsilon \rightarrow 0$ for a.e. $x \in \mathbb{R}_{-}^{n}$. Letting $\epsilon \rightarrow 0$ in (47), with (25), Sobolev's inequality and since $\mu_{\epsilon}^{p_{\epsilon}} \leq 1$ for all $\epsilon>0$ small enough, we get that there exists a constant $C>0$ independant of $R$ such that

$$
\int_{\mathbb{R}_{-}^{n}}\left|\nabla v_{R}\right|^{2} d x \leq C_{\alpha}\left\|\nabla \eta_{R}\right\|_{n}^{2} \cdot C+(1+\alpha) \cdot C
$$

for all $R>0$. Since $\left\|\nabla \eta_{R}\right\|_{n}^{2}=\left\|\nabla \eta_{1}\right\|_{n}^{2}$ for all $R>0$, we get that there exists $C>0$ independant of $R$ such that

$$
\int_{\mathbb{R}_{-}^{n}}\left|\nabla v_{R}\right|^{2} d x \leq C
$$

for all $R>0$. It then follows that there exists $v \in H_{1,0}^{2}\left(\mathbb{R}_{-}^{n}\right)$ such that $v_{R} \rightarrow v$ weakly in $H_{1,0}^{2}\left(\mathbb{R}_{-}^{n}\right)$ when $R \rightarrow+\infty$ and $v_{R}(x) \rightarrow v(x)$ when $R \rightarrow+\infty$ for a.e. $x \in \mathbb{R}_{-}^{n}$. As easily checked, we then obtain that $v_{\eta}=\eta v$ (we omit the proof of this fact. It is straightforward). This ends Step 5.6.

Step 5.7: We claim that

$$
v \not \equiv 0
$$

Indeed, we let $R>0$. We proceed as in Case 5.2.2 of the proof of (31) in Step 5.2, for any $i, j=1, \ldots, n$, we let $\left(\tilde{g}_{\epsilon}\right)_{i j}=\left(\partial_{i} \varphi\left(k_{\epsilon} x\right), \partial_{j} \varphi\left(k_{\epsilon} x\right)\right)$, where $(\cdot, \cdot)$ denotes the Euclidean scalar product on $\mathbb{R}^{n}$. We consider $\tilde{g}_{\epsilon}$ as a metric on $\mathbb{R}^{n}$. We let

$$
\Delta_{\tilde{g}_{\epsilon}}=-\tilde{g}_{\epsilon}^{i j}\left(\partial_{i j}-\Gamma_{i j}^{k}\left(\tilde{g}_{\epsilon}\right) \partial_{k}\right)
$$

where $\tilde{g}_{\epsilon}^{i j}:=\left(\tilde{g}_{\epsilon}^{-1}\right)_{i j}$ are the coordinates of the inverse of the tensor $\tilde{g}_{\epsilon}$ and the $\Gamma_{i j}^{k}\left(\tilde{g}_{\epsilon}\right)$ 's are the Christoffel symbols of the metric $\tilde{g}_{\epsilon}$. With a change of variable and the definition (45), equation (23) rewrites as

$$
\Delta_{\tilde{g}_{\epsilon}}\left(\eta_{R} v_{\epsilon}\right)+k_{\epsilon}^{2} a_{\epsilon} \circ \varphi\left(k_{\epsilon} x\right) \eta_{R} v_{\epsilon}=\frac{\left(\eta_{R} v_{\epsilon}\right)^{2^{\star}-1-p_{\epsilon}}}{\left|\frac{\varphi\left(k_{\epsilon} x\right)}{k_{\epsilon}}\right|^{s}} \text { in } \mathcal{D}^{\prime}\left(B_{R}(0) \cap\left\{x_{1}<0\right\}\right)
$$

for all $\epsilon>0$. With (27), (45) and since $s \in(0,2)$, we get that $0 \leq v_{\epsilon} \leq 1$ and that there exists $p>\frac{n}{2}$ such that the RHS of (48) is bounded in $L^{p}$ when $\epsilon \rightarrow 0$. It then follows from standard elliptic theory that there exists $\alpha>0$ such that

$$
\left\|\eta_{R} v_{\epsilon}\right\|_{C^{0, \alpha}\left(B_{R / 2}(0) \cap\left\{x_{1} \leq 0\right\}\right)}=O(1)
$$

when $\epsilon \rightarrow 0$. It then follows from Ascoli's theorem that for any $\alpha^{\prime} \in(0, \alpha)$, $v_{R} \in C^{0, \alpha^{\prime}}\left(B_{R / 2}(0) \cap\left\{x_{1} \leq 0\right\}\right)$ and that, up to a subsequence,

$$
\lim _{\epsilon \rightarrow 0} \eta_{R} v_{\epsilon}=v_{R} \text { in } C^{0, \alpha^{\prime}}\left(B_{R / 4}(0) \cap\left\{x_{1} \leq 0\right\}\right)
$$

With (45) and (43), we have that $\left(\eta_{R} v_{\epsilon}\right)\left(-\lambda_{\epsilon}, \theta_{\epsilon}\right)=1$ for all $\epsilon>0$ and $R>0$ large enough. Passing to the limit $\epsilon \rightarrow 0$ in this last equality, using (49) and (44), we get that

$$
v_{R}\left(-\lambda_{0}, \theta_{0}, 0\right)=1
$$


for $R>0$ large enough. With the same type of arguments, we get that $v \in$ $C^{0, \alpha}\left(\left\{x_{1} \leq 0\right\}\right)$ and that $\lim _{R \rightarrow+\infty} v_{R}=v$ in $C_{l o c}^{0, \alpha}\left(\left\{x_{1} \leq 0\right\}\right)$. Since $\eta_{R} v=v_{R}$, we get that $v\left(-\lambda_{0}, \theta_{0}\right)=1$. In particular, $v \not \equiv 0$ and $\lambda_{0}>0$. This ends Step 5.7.

Step 5.8: We claim that there exists $\theta \in(0,1)$ such that $v \in C^{1, \theta}\left(\overline{\mathbb{R}_{-}^{n}}\right)$ and

$$
v_{\epsilon} \rightarrow v \text { in } C_{l o c}^{1, \theta}\left(\overline{\mathbb{R}_{-}^{n}}\right)
$$

when $\epsilon \rightarrow 0$. Indeed, it follows from Step 5.7 that there exists $\alpha>0$ such that for all $R>0$, there exists $C(R)>0$ such that

$$
\left\|v_{\epsilon}\right\|_{C^{0, \alpha}\left(B_{R}(0) \cap\left\{x_{1} \leq 0\right\}\right)} \leq C(R) .
$$

Following the proof of Proposition [8.1] we let

$$
\alpha_{0}:=\sup \left\{\alpha \in(0,1) / \forall R>0, \exists C(R)>0 \text { s.t. }\left\|v_{\epsilon}\right\|_{C^{0, \alpha}\left(B_{R}(0) \cap\left\{x_{1} \leq 0\right\}\right)} \leq C(R)\right\} .
$$

We let $\alpha \in\left(0, \alpha_{0}\right)$ and $R>0$. We let $\tilde{R}>R$. There exists $C(\tilde{R})>0$ such that

$$
\left\|v_{\epsilon}\right\|_{C^{0, \alpha}\left(B_{\tilde{R}}(0) \cap\left\{x_{1} \leq 0\right\}\right)} \leq C(\tilde{R}) .
$$

Since $v_{\epsilon} \equiv 0$ on $\partial \mathbb{R}_{-}^{n}$, we get with (50) that

$$
\left|v_{\epsilon}(x)\right|=\left|v_{\epsilon}(x)-v_{\epsilon}\left(x-\left(x_{1}, 0\right)\right)\right| \leq C(\tilde{R})\left|x_{1}\right|^{\alpha}
$$

for all $B_{\tilde{R}}(0) \cap\left\{x_{1}<0\right\}$ and all $\epsilon>0$. It then follows from the properties of $\varphi$ (see (16) with $x_{0}=0$ ) that

$$
0 \leq f_{\epsilon}(x):=\frac{\left(\eta v_{\epsilon}\right)^{2^{\star}-1-p_{\epsilon}}}{\left|\frac{\varphi\left(k_{\epsilon} x\right)}{k_{\epsilon}}\right|^{S}} \leq \frac{C}{|x|^{s-\left(2^{\star}-1-p_{\epsilon}\right) \alpha}}
$$

for all $\epsilon>0$ and all $x \in B_{\tilde{R}}(0) \cap\left\{x_{1}<0\right\}$. With the properties (16), we get that for any $\tilde{R}>0$ and any $p>1$, we have that

$$
\int_{B_{\tilde{R}}(0) \cap\left\{x_{1}<0\right\}} \frac{d x}{\left|\frac{\varphi\left(k_{\epsilon} x\right)}{k_{\epsilon}}\right|^{p}} \leq C \int_{B_{\tilde{R}}(0)} \frac{d x}{|x|^{p}}
$$

for all $\epsilon>0$ (note that the RHS can be infinite). Using the same strategy as in the proof of Proposition 8.1 we get that there exists $\theta \in(0,1)$ such that $v \in C^{1, \theta}\left(\overline{\mathbb{R}_{-}^{n}}\right)$ and

$$
v_{\epsilon} \rightarrow v \text { in } C_{l o c}^{1, \theta}\left(\overline{\mathbb{R}_{-}^{n}}\right)
$$

when $\epsilon \rightarrow 0$. We omit the proof and refer to the proof of Proposition 8.1 for the details. This ends Step 5.8.

Step 5.9: We claim that

$$
\Delta v=\frac{v^{2^{\star}-1}}{|x|^{s}} \text { in } \mathcal{D}^{\prime}\left(\mathbb{R}_{-}^{n}\right)
$$

and that

$$
\int_{\mathbb{R}_{-}^{n}}|\nabla v|^{2} d x=\mu_{s, a}(\Omega)^{\frac{2^{\star}}{2^{\star}-2}}=\mu_{s}\left(\mathbb{R}_{-}^{n}\right)^{\frac{2^{\star}}{2^{\star}-2}} .
$$

Indeed, passing to the weak limit $\epsilon \rightarrow 0$ and then to the weak limit $R \rightarrow+\infty$ in (48), we get that

$$
\Delta v=\frac{v^{2^{\star}-1}}{|x|^{s}} \text { in } \mathcal{D}^{\prime}\left(\mathbb{R}_{-}^{n}\right) .
$$


Testing this equality with $v \in H_{1,0}^{2}\left(\mathbb{R}_{-}^{n}\right) \backslash\{0\}$ and using the optimal Hardy-Sobolev inequality (2), we get that

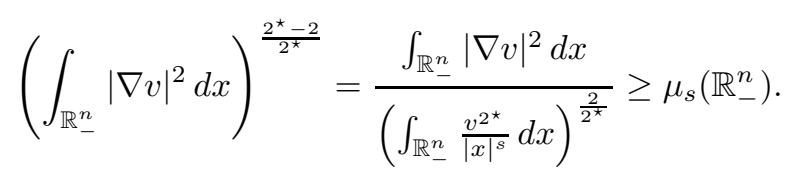

We then obtain that

$$
\int_{\mathbb{R}_{-}^{n}}|\nabla v|^{2} d x \geq \mu_{s}\left(\mathbb{R}_{-}^{n}\right)^{\frac{2^{\star}}{2^{\star}-2}}
$$

Since $0 \leq v_{\epsilon} \leq 1$, it follows from Lebesgue's theorem that $v_{\epsilon} \rightarrow v$ strongly in $L_{l o c}^{\frac{2 n}{n-2}}\left(\mathbb{R}_{-}^{n} \cap\left\{x_{1} \leq 0\right\}\right)$ when $\epsilon \rightarrow 0$. Passing to the weak limit in (46) and using (24), we get that

$$
\begin{aligned}
\int_{\mathbb{R}_{-}^{n}}\left|\nabla v_{R}\right|^{2} d x \leq & C_{\alpha}\left\|\nabla \eta_{R}\right\|_{n}^{2} \cdot\|v\|_{L^{\frac{2 n}{n-2}}}^{2}\left(B_{2 R}(0) \backslash B_{R}(0)\right) \\
& +(1+\alpha) \cdot\left(\lim _{\epsilon \rightarrow 0} \frac{p_{\epsilon}(n-2)}{\mu^{2^{\star}-2}}\right) \mu_{s, a}(\Omega)^{\frac{2^{\star}}{2^{\star}-2}}
\end{aligned}
$$

for all $R>0$. Since $v \in H_{1,0}^{2}\left(\mathbb{R}_{-}^{n}\right)$, it follows from Sobolev's theorem that $v \in$ $L^{\frac{2 n}{n-2}}\left(\mathbb{R}_{-}^{n}\right)$. Since $\left\|\nabla \eta_{R}\right\|_{n}^{2}=\left\|\nabla \eta_{1}\right\|_{n}^{2}$ is independant of $R>0$ and $v \in L^{\frac{2 n}{n-2}}\left(\mathbb{R}_{-}^{n}\right)$, letting $R \rightarrow+\infty$ in (55), we get that

$$
\int_{\mathbb{R}_{-}^{n}}|\nabla v|^{2} d x \leq(1+\alpha) \cdot\left(\lim _{\epsilon \rightarrow 0} \mu_{\epsilon}^{\frac{p_{\epsilon}(n-2)}{2^{\star}-2}}\right) \mu_{s, a}(\Omega)^{\frac{2^{\star}}{2^{\star}-2}}
$$

Since $\alpha>0$ is arbitrary and $\mu_{\epsilon} \leq 1$, we get with (54), (56), Proposition 3.1 and (53) that

$$
\int_{\mathbb{R}_{-}^{n}}|\nabla v|^{2} d x=\mu_{s, a}(\Omega)^{\frac{2^{\star}}{2^{\star}-2}}=\mu_{s}\left(\mathbb{R}_{-}^{n}\right)^{\frac{2^{\star}}{2^{\star}-2}},
$$

and that

$$
\lim _{\epsilon \rightarrow 0} \mu_{\epsilon}^{p_{\epsilon}}=1 .
$$

This ends Step 5.9. Proposition [5.1 then follows from Steps 5.1 to 5.9.

Step 5.10: We claim that under the hypothesis of Proposition 5.1 we have that

$$
\lim _{R \rightarrow+\infty} \lim _{\epsilon \rightarrow 0} \int_{\Omega \backslash B_{R k_{\epsilon}}(0)} \frac{u_{\epsilon}^{2^{\star}-p_{\epsilon}}}{|x|^{s}} d x=0 .
$$

Indeed, we let $R>0$. Since $D_{0} \varphi=I d_{\mathbb{R}^{n}}$ and $\varphi(0)=0$, we have that

$$
\varphi\left(B_{\frac{R}{2} k_{\epsilon}}(0)\right) \subset B_{R k_{\epsilon}}(0)
$$


for all $R>0$ and $\epsilon>0$ small enough. With a change of variable and (24), we get that

$$
\begin{aligned}
& \int_{\Omega \backslash B_{R k_{\epsilon}}(0)} \frac{u_{\epsilon}^{2^{\star}-p_{\epsilon}}}{|x|^{s}} d x \leq \int_{\Omega \backslash \varphi\left(B_{\frac{R}{2} k_{\epsilon}}(0)\right)} \frac{u_{\epsilon}^{2^{\star}-p_{\epsilon}}}{|x|^{s}} d x \\
& \leq \int_{\Omega} \frac{u_{\epsilon}^{2^{\star}-p_{\epsilon}}}{|x|^{s}} d x-\int_{\varphi\left(B_{\frac{R}{2} k_{\epsilon}}(0)\right)} \frac{u_{\epsilon}^{2^{\star}-p_{\epsilon}}}{|x|^{s}} d x \\
& \leq \mu_{s, a}(\Omega)^{\frac{2^{\star}}{2^{\star}-2}}+o(1)-\mu_{\epsilon}^{-p_{\epsilon} \frac{(n-2)^{2}}{2(2-s)}}(1+o(1)) \int_{B_{\frac{R}{2}}(0)} \frac{v_{\epsilon}^{2^{\star}-p_{\epsilon}}}{|x|^{s}} d x .
\end{aligned}
$$

when $\epsilon \rightarrow 0$. Letting $\epsilon \rightarrow 0$ and then $R \rightarrow+\infty$, we get with (29) and Proposition 5.1 that

$$
\begin{aligned}
\lim _{R \rightarrow+\infty} \lim _{\epsilon \rightarrow 0} \int_{\Omega \backslash B_{R k_{\epsilon}}(0)} \frac{u_{\epsilon}^{2^{\star}-p_{\epsilon}}}{|x|^{s}} d x & \leq \mu_{s, a}(\Omega)^{\frac{2^{\star}}{2^{\star}-2}}-\lim _{R \rightarrow+\infty} \int_{B_{\frac{R}{2}}(0)} \frac{v^{2^{\star}}}{|x|^{s}} d x \\
& \leq \mu_{s, a}(\Omega)^{\frac{2^{\star}}{2^{\star}-2}}-\int_{\mathbb{R}_{-}^{n}} \frac{v^{2^{\star}}}{|x|^{s}} d x=0
\end{aligned}
$$

This last inequality yields (57).

\section{ReFined Blow-Up anALysis and STRONG POINTWise ESTIMATES}

The objective of this section is the proof of the following strong pointwise estimate

Proposition 6.1. Let $\Omega$ be a smooth bounded domain of $\mathbb{R}^{n}, n \geq 3$. We let $s \in(0,2)$. We let $\left(p_{\epsilon}\right)_{\epsilon>0}$ such that $p_{\epsilon} \in\left[0,2^{\star}-2\right)$ for all $\epsilon>0$ and (22) holds. We consider $\left(u_{\epsilon}\right)_{\epsilon>0} \in H_{1,0}^{2}(\Omega)$ such that (14), (15), (23), (24) and (26) hold. We let $\mu_{\epsilon}$ as in 27). Then, there exists $C>0$ such that

$$
u_{\epsilon}(x) \leq C \cdot\left(\frac{\mu_{\epsilon}}{\mu_{\epsilon}^{2}+|x|^{2}}\right)^{\frac{n-2}{2}}
$$

for all $\epsilon>0$ and all $x \in \Omega$.

This type of strong pointwise estimate first appeared in 21] in the Euclidean context, and in 23] in the Riemannian context. General estimates are in [10.

Proof. The rest of the section is mainly devoted to the proof of the proposition. Here again, we follows the strategy of [10. We let $\left(u_{\epsilon}\right)_{\epsilon>0}$ satisfying the hypothesis of Proposition 6.1

Step 6.1: We claim that there exists $C>0$ such that

$$
|x|^{\frac{n-2}{2}} u_{\epsilon}(x)^{1-\frac{p_{\epsilon}}{2^{\star}-2}} \leq C
$$

for all $\epsilon>0$ and all $x \in \Omega$.

We proceed by contradiction and let $y_{\epsilon} \in \Omega$ such that

$$
\left|y_{\epsilon}\right|^{\frac{n-2}{2}} u_{\epsilon}\left(y_{\epsilon}\right)^{1-\frac{p_{\epsilon}}{2^{\star}-2}}=\sup _{x \in \Omega}|x|^{\frac{n-2}{2}} u_{\epsilon}(x)^{1-\frac{p_{\epsilon}}{2^{\star}-2}} \rightarrow+\infty
$$

when $\epsilon \rightarrow 0$. We let

$$
\nu_{\epsilon}:=u_{\epsilon}\left(y_{\epsilon}\right)^{-\frac{2}{n-2}} \text { and } \ell_{\epsilon}:=\nu_{\epsilon}^{1-\frac{p_{\epsilon}}{2^{\star}-2}}
$$


for all $\epsilon>0$. It follows from (60) and (61) that

$$
\lim _{\epsilon \rightarrow 0} \nu_{\epsilon}=0 \text { and } \lim _{\epsilon \rightarrow 0} \frac{\left|y_{\epsilon}\right|}{\ell_{\epsilon}}=+\infty .
$$

It follows from (27) and (29) that

$$
\lim _{\epsilon \rightarrow 0} \nu_{\epsilon}^{p_{\epsilon}}=1
$$

We let

$$
\gamma_{\epsilon}^{2}:=\left|y_{\epsilon}\right|^{s}\left|u_{\epsilon}\left(y_{\epsilon}\right)\right|^{-\left(2^{*}-2-p_{\epsilon}\right)},
$$

for all $\epsilon>0$. It follows from (62) that

$$
\lim _{\epsilon \rightarrow 0} \frac{\gamma_{\epsilon}}{\left|y_{\epsilon}\right|}=0
$$

Case 6.1.1: We assume that, up to a subsequence, there exists $\rho>0$ such that

$$
\frac{d\left(y_{\epsilon}, \partial \Omega\right)}{\gamma_{\epsilon}} \geq 3 \rho
$$

for all $\epsilon>0$. For any $x \in B_{2 \rho}(0)$ and any $\epsilon>0$, we let

$$
w_{\epsilon}(x):=\nu_{\epsilon}^{\frac{n-2}{2}} u_{\epsilon}\left(y_{\epsilon}+\gamma_{\epsilon} x\right)
$$

Note that $w_{\epsilon}$ is well defined thanks to (66). With (60) and (64), we get that

$$
\left|\frac{y_{\epsilon}}{\left|y_{\epsilon}\right|}+\frac{\gamma_{\epsilon}}{\left|y_{\epsilon}\right|} x\right|^{\frac{n-2}{2}} w_{\epsilon}(x)^{1-\frac{p_{\epsilon}}{2^{\star}-2}} \leq 1
$$

In particular, with (62), there exists $C_{0}>0$ such that

$$
0 \leq w_{\epsilon}(x) \leq C_{0}
$$

for all $x \in B_{2 \rho}(0)$ and all $\epsilon>0$. With (23), we get that

$$
\Delta w_{\epsilon}+\gamma_{\epsilon}^{2} a_{\epsilon}\left(y_{\epsilon}+\gamma_{\epsilon} x\right) w_{\epsilon}=\frac{w_{\epsilon}^{2^{\star}-1-p_{\epsilon}}}{\left|\frac{y_{\epsilon}}{\left|y_{\epsilon}\right|}+\frac{\gamma_{\epsilon}}{\left|y_{\epsilon}\right|} x\right|^{s}}
$$

for all $x \in B_{2 \rho}(0)$ and all $\epsilon>0$. Since (62) and (68) hold, it follows from standard elliptic theory that there exists $w \in C^{1}\left(B_{2 \rho}(0)\right)$ such that $w \geq 0$ and

$$
\lim _{\epsilon \rightarrow 0} w_{\epsilon}=w
$$

in $C_{l o c}^{1}\left(B_{2 \rho}(0)\right)$. It follows from (67) that $w(0)=1$. With a change of variable, we get that

$$
\int_{B_{\rho \gamma_{\epsilon}}\left(y_{\epsilon}\right)} \frac{u_{\epsilon}(x)^{2^{\star}-p_{\epsilon}}}{|x|^{s}} d x=\frac{\gamma_{\epsilon}^{n} u_{\epsilon}\left(y_{\epsilon}\right)^{2^{\star}-p_{\epsilon}}}{\left|y_{\epsilon}\right|^{s}} \int_{B_{\rho}(0)} \frac{w_{\epsilon}(x)^{2^{\star}-p_{\epsilon}}}{\left|\frac{y_{\epsilon}}{\left|y_{\epsilon}\right|}+\frac{\gamma_{\epsilon}}{\left|y_{\epsilon}\right|} \cdot x\right|^{s}} d x .
$$

With (64), 63), 62) and (61), we then get that

$$
\frac{\gamma_{\epsilon}^{n} u_{\epsilon}\left(y_{\epsilon}\right)^{2^{\star}-p_{\epsilon}}}{\left|y_{\epsilon}\right|^{s}}=(1+o(1)) \cdot\left(\frac{\left|y_{\epsilon}\right|}{\ell_{\epsilon}}\right)^{\frac{s(n-2)}{2}} \rightarrow+\infty
$$

when $\epsilon \rightarrow 0$. With (70), (69) and (24), we get that

$$
\int_{B_{\rho}(0)} w^{2^{\star}} d x=0
$$

and then $w \equiv 0$. A contradiction since $w(0)=1$. This ends Case 6.1.1. 
Case 6.1.2: We assume that

$$
\lim _{\epsilon \rightarrow 0} \frac{d\left(y_{\epsilon}, \partial \Omega\right)}{\gamma_{\epsilon}}=0 .
$$

It then follows that there exists $y_{0} \in \partial \Omega$ such that

$$
\lim _{\epsilon \rightarrow 0} y_{\epsilon}=y_{0} .
$$

Since $y_{0} \in \partial \Omega$, which is smooth, we let $\varphi: U \rightarrow V$ as in (16) with $x_{0}=y_{0}$ and where $U, V$ are open neighborhoods of 0 and $y_{0}$ respectively. We let $\tilde{u}_{\epsilon}=u_{\epsilon} \circ \varphi$, which is defined on $U \cap\left\{x_{1} \leq 0\right\}$. For any $i, j=1, \ldots, n$, we let $g_{i j}=\left(\partial_{i} \varphi, \partial_{j} \varphi\right)$, where $(\cdot, \cdot)$ denotes the Euclidean scalar product on $\mathbb{R}^{n}$, and we consider $g$ as a metric on $\mathbb{R}^{n}$. We let $\Delta_{g}=-d i v_{g}(\nabla)$ the Laplace-Beltrami operator with respect to the metric $g$. In the basis we choose,

$$
\Delta_{g}=-g^{i j}\left(\partial_{i j}-\Gamma_{i j}^{k} \partial_{k}\right)
$$

where $g^{i j}=\left(g^{-1}\right)_{i j}$ are the coordinates of the inverse of the tensor $g$ and the $\Gamma_{i j}^{k}$ 's are the Christoffel symbols of the metric $g$. As easily checked, we have that

$$
\Delta_{g} \tilde{u}_{\epsilon}+a_{\epsilon} \circ \varphi(x) \cdot \tilde{u}_{\epsilon}=\frac{\tilde{u}_{\epsilon}^{2^{\star}-1-p_{\epsilon}}}{|\varphi(x)|^{s}}
$$

weakly in $U \cap\left\{x_{1}<0\right\}$. We let $z_{\epsilon} \in \partial \Omega$ such that

$$
\left|z_{\epsilon}-y_{\epsilon}\right|=d\left(y_{\epsilon}, \partial \Omega\right)
$$

We let $\tilde{y}_{\epsilon}, \tilde{z}_{\epsilon} \in U$ such that

$$
\varphi\left(\tilde{y}_{\epsilon}\right)=y_{\epsilon} \text { and } \varphi\left(\tilde{z}_{\epsilon}\right)=z_{\epsilon}
$$

It follows from the properties of $\varphi$ that

$$
\lim _{\epsilon \rightarrow 0} \tilde{y}_{\epsilon}=\lim _{\epsilon \rightarrow 0} \tilde{z}_{\epsilon}=0,\left(\tilde{y}_{\epsilon}\right)_{1}<0 \text { and }\left(\tilde{z}_{\epsilon}\right)_{1}=0
$$

At last, we let

$$
\tilde{w}_{\epsilon}(x):=\frac{\tilde{u}_{\epsilon}\left(\tilde{z}_{\epsilon}+\gamma_{\epsilon} x\right)}{\tilde{u}_{\epsilon}\left(\tilde{y}_{\epsilon}\right)}
$$

for all $x \in \frac{U-\tilde{z}_{\epsilon}}{\gamma_{\epsilon}} \cap\left\{x_{1}<0\right\}$. With (74), we get that $\tilde{w}_{\epsilon}$ is defined on $B_{R}(0) \cap\left\{x_{1}<0\right\}$ for all $R>0$, as soon as $\epsilon$ is small enough. The function $\tilde{w}_{\epsilon}$ verifies

$$
\Delta_{\tilde{g}_{\epsilon}} \tilde{w}_{\epsilon}+\gamma_{\epsilon}^{2} a_{\epsilon} \circ \varphi\left(\tilde{z}_{\epsilon}+\gamma_{\epsilon} x\right) \tilde{w}_{\epsilon}=\frac{\tilde{w}_{\epsilon}^{2^{\star}-1-p_{\epsilon}}}{\left|\frac{\varphi\left(\tilde{z}_{\epsilon}+\gamma_{\epsilon} x\right)}{\left|y_{\epsilon}\right|}\right|^{s}}
$$

weakly in $B_{R}(0) \cap\left\{x_{1}<0\right\}$. In this expression, $\tilde{g}_{\epsilon}=g\left(\tilde{z}_{\epsilon}+\gamma_{\epsilon} x\right)$ and $\Delta_{\tilde{g}_{\epsilon}}$ is the Laplace-Beltrami operator with respect to the metric $\tilde{g}_{\epsilon}$. With (71), (72) and (73), we get that

$$
\varphi\left(\tilde{z}_{\epsilon}+\gamma_{\epsilon} x\right)=y_{\epsilon}+O_{R}(1) \gamma_{\epsilon}
$$

for all $x \in B_{R}(0) \cap\left\{x_{1} \leq 0\right\}$ and all $\epsilon>0$, where there exists $C_{R}>0$ such that $\left|O_{R}(1)\right| \leq C_{R}$ for all $x \in B_{R}(0) \cap\left\{x_{1}<0\right\}$. With (65), we then get that

$$
\lim _{\epsilon \rightarrow 0} \frac{\left|\varphi\left(\tilde{z}_{\epsilon}+\gamma_{\epsilon} x\right)\right|}{\left|y_{\epsilon}\right|}=1
$$

in $C^{0}\left(B_{R}(0) \cap\left\{x_{1} \leq 0\right\}\right)$. It then follows that

$$
\Delta_{\tilde{g}_{\epsilon}} \tilde{w}_{\epsilon}+\gamma_{\epsilon}^{2} a_{\epsilon} \circ \varphi\left(\tilde{z}_{\epsilon}+\gamma_{\epsilon} x\right) \tilde{w}_{\epsilon}=(1+o(1)) \tilde{w}_{\epsilon}^{2^{\star}-1-p_{\epsilon}}
$$


weakly in $B_{R}(0) \cap\left\{x_{1}<0\right\}$, where $\lim _{\epsilon \rightarrow 0} o(1)=0$ in $C^{0}\left(B_{R}(0) \cap\left\{x_{1} \leq 0\right\}\right)$. Since $\tilde{w}_{\epsilon}$ vanishes on $B_{R}(0) \cap\left\{x_{1}=0\right\}$ (in the sense of the trace) and that $0 \leq \tilde{w}_{\epsilon} \leq 2$ (see for instance the proof of (68) ), it follows from standard elliptic theory that there exists $\tilde{w} \in C^{1}\left(B_{R}(0) \cap\left\{x_{1} \leq 0\right\}\right)$ such that

$$
\lim _{\epsilon \rightarrow 0} \tilde{w}_{\epsilon}=\tilde{w}
$$

in $C^{0}\left(B_{\frac{R}{2}}(0) \cap\left\{x_{1} \leq 0\right\}\right)$. In particular,

$$
\tilde{w} \equiv 0 \text { on } B_{\frac{R}{2}}(0) \cap\left\{x_{1}=0\right\} .
$$

Moreover, it follows from (71), (172) and (73) that

$$
\tilde{w}_{\epsilon}\left(\frac{\tilde{y}_{\epsilon}-\tilde{z}_{\epsilon}}{\gamma_{\epsilon}}\right)=1 \text { and } \lim _{\epsilon \rightarrow 0} \frac{\tilde{y}_{\epsilon}-\tilde{z}_{\epsilon}}{\gamma_{\epsilon}}=0 .
$$

In particular, $\tilde{w}(0)=1$. A contradiction with (75). This ends Case 6.1.2.

In both cases, we have contradicted (60). This proves (59) and ends Step 6.1.

As a remark, it follows from (23), (26), (159) and standard elliptic theory that

$$
\lim _{\epsilon \rightarrow 0} u_{\epsilon}=0 \text { in } C_{l o c}^{2}(\bar{\Omega} \backslash\{0\}) .
$$

Step 6.2: This step is a slight improvement of (59). We claim that

$$
\lim _{R \rightarrow+\infty} \lim _{\epsilon \rightarrow 0} \sup _{x \in \Omega \backslash B_{R k_{\epsilon}}(0)}|x|^{\frac{n-2}{2}} u_{\epsilon}(x)^{1-\frac{p_{\epsilon}}{2^{\star}-2}}=0 .
$$

We proceed by contradiction and assume that there exists $\epsilon_{0}>0$ and a family $\left(y_{\epsilon}\right)_{\epsilon>0} \in \Omega$ such that

$$
\left|y_{\epsilon}\right|^{\frac{n-2}{2}} u_{\epsilon}\left(y_{\epsilon}\right)^{1-\frac{p_{\epsilon}}{2^{\star}-2}} \geq \epsilon_{0} \text { and } \lim _{\epsilon \rightarrow 0} \frac{\left|y_{\epsilon}\right|}{k_{\epsilon}}=+\infty .
$$

We let

$$
\nu_{\epsilon}:=u_{\epsilon}\left(y_{\epsilon}\right)^{-\frac{2}{n-2}} \text { and } \gamma_{\epsilon}:=\nu_{\epsilon}^{1-\frac{p_{\epsilon}}{2^{\star}-2}}
$$

for all $\epsilon>0$. It follows from (76), (59), (78) and (79) that there exists $\rho_{0} \in \mathbb{R}$ such that

$$
\lim _{\epsilon \rightarrow 0} y_{\epsilon}=0, \lim _{\epsilon \rightarrow 0} \nu_{\epsilon}=0 \text { and } \lim _{\epsilon \rightarrow 0} \frac{\left|y_{\epsilon}\right|}{\gamma_{\epsilon}}=\rho_{0}>0 .
$$

Note that it follows from (27) and (29) that

$$
\lim _{\epsilon \rightarrow 0} \nu_{\epsilon}^{p_{\epsilon}}=1 \text {. }
$$

We let $\varphi: U \rightarrow V$ as in (16) with $x_{0}=0$ and where $U, V$ are open neighborhoods of 0 . For any $x \in \frac{U}{\gamma_{\epsilon}} \cap\left\{x_{1}<0\right\}$, we let

$$
\bar{w}_{\epsilon}(x):=\nu_{\epsilon}^{\frac{n-2}{2}} u_{\epsilon} \circ \varphi\left(\gamma_{\epsilon} x\right) .
$$

It follows from (59) and the properties (16) of $\varphi$ that there exists $C>0$ such that

$$
|x|^{\frac{n-2}{2}} \bar{w}_{\epsilon}(x)^{1-\frac{p_{\epsilon}}{2^{x}-2}} \leq C
$$

for all $x \in \frac{U}{\gamma_{\epsilon}} \cap\left\{x_{1}<0\right\}$ and all $\epsilon>0$. As above, we let the metric $\left(\bar{g}_{\epsilon}\right)_{i j}=$ $\left(\partial_{i} \varphi, \partial_{j} \varphi\right)\left(\gamma_{\epsilon} x\right)$ for $i, j=1, \ldots, n$. With (23), we get that

$$
\Delta_{\bar{g}_{\epsilon}} \bar{w}_{\epsilon}+\gamma_{\epsilon}^{2} a_{\epsilon} \circ \varphi\left(\gamma_{\epsilon} x\right) \bar{w}_{\epsilon}=\frac{\bar{w}_{\epsilon}^{2^{\star}-1-p_{\epsilon}}}{\left|\frac{\varphi\left(\gamma_{\epsilon} x\right)}{\gamma_{\epsilon}}\right|^{s}}
$$


in $\frac{U}{\gamma_{\epsilon}} \cap\left\{x_{1}<0\right\}$ for all $\epsilon>0$. Moreover, $\bar{w}_{\epsilon}$ vanishes on $\frac{U}{\gamma_{\epsilon}} \cap\left\{x_{1}=0\right\}$. It then follows from (83), (84) and standard elliptic theory (see for instance 20]) that there exists $\left.\bar{w} \in C^{0}\left(\mathbb{R}_{-}^{n} \cap\left\{x_{1}=0\right\}\right) \backslash\{0\}\right)$ such that $\bar{w} \geq 0$ and

$$
\lim _{\epsilon \rightarrow 0} \bar{w}_{\epsilon}=\bar{w}
$$

in $\left.C^{0}\left(\mathbb{R}_{-}^{n} \cap\left\{x_{1}=0\right\}\right) \backslash\{0\}\right)$. We now write $y_{\epsilon}=\varphi\left(\gamma_{\epsilon} \tilde{y}_{\epsilon}\right)$. It follows from (80) that $\lim _{\epsilon \rightarrow 0}=y_{0} \neq 0$. As a consequence,

$$
\bar{w}\left(y_{0}\right)=\lim _{\epsilon \rightarrow 0} \bar{w}_{\epsilon}\left(\tilde{y}_{\epsilon}\right)=1,
$$

and then $\bar{w} \not \equiv 0$. We let $0<\delta<R$. With a change of variable, we have that

$$
\lim _{\epsilon \rightarrow 0} \int_{\varphi\left(B_{R \gamma_{\epsilon}}(0)\right) \backslash \varphi\left(B_{\delta \gamma_{\epsilon}}(0)\right)} \frac{u_{\epsilon}(x)^{2^{\star}-p_{\epsilon}}}{|x|^{s}} d x=\int_{B_{R}(0) \backslash B_{\delta}(0)} \frac{\bar{w}(x)^{2^{\star}}}{|x|^{s}} d x .
$$

With (78), we get that for any $\rho>0$, we have that

$$
B_{\rho k_{\epsilon}}(0) \cap\left(\varphi\left(B_{R \gamma_{\epsilon}}(0)\right) \backslash \varphi\left(B_{\delta \gamma_{\epsilon}}(0)\right)\right)=\emptyset
$$

for all $\epsilon>0$ small enough, up to a subsequence. It then follows from (57) that

$$
\lim _{\epsilon \rightarrow 0} \int_{\varphi\left(B_{R \gamma_{\epsilon}}(0)\right) \backslash \varphi\left(B_{\delta \gamma_{\epsilon}}(0)\right)} \frac{u_{\epsilon}(x)^{2^{\star}-p_{\epsilon}}}{|x|^{s}} d x=0 .
$$

This equality and (85) yield

$$
\int_{B_{R}(0) \backslash B_{\delta}(0)} \frac{\bar{w}^{2^{\star}}}{|x|^{s}} d x=0
$$

for all $R>\delta>0$. We then get that $\bar{w} \equiv 0$. A contradiction since $\bar{w}\left(y_{0}\right)=1$. This ends Step 6.2.

Step 6.3: We prove a first approximation of (58). More precisely, we claim that for any $\alpha \in(0, n-2)$, there exists $C_{\alpha}>0$ such that

$$
|x|^{\alpha} \mu_{\epsilon}^{\frac{n-2}{2}-\alpha} u_{\epsilon}(x) \leq C_{\alpha}
$$

for all $\epsilon>0$ and all $x \in \Omega$. Indeed, since $\Delta+a$ is coercive on $\Omega$ and $\left(a_{\epsilon}\right)_{\epsilon>0}$ satisfies (14) and (15), there exists $U_{0}$ an open subset of $\mathbb{R}^{n}$ such that $\bar{\Omega} \subset \subset U_{0}$, there exists $\alpha_{0}>0$ and there exists $\lambda>0$ such that

$$
\int_{U_{0}}\left(|\nabla \varphi|^{2}+\left(a_{\epsilon}-2 \alpha_{0}\right) \varphi^{2}\right) d x \geq \lambda \int_{U_{0}} \varphi^{2} d x
$$

for all $\varphi \in C_{c}^{1}\left(U_{0}\right)$ and all $\epsilon>0$. In other words, the family of the operators $\Delta+a_{\epsilon}-\alpha_{0}$ is uniformly coercive in a neighborhood of $\bar{\Omega}$. We let $G_{\epsilon} \in C^{2}\left(U_{0} \times U_{0} \backslash\right.$ $\left.\left\{(x, x) / x \in U_{0}\right\}\right)$ be the Green's function for $\Delta+a_{\epsilon}-\alpha_{0}$ with Dirichlet condition in $U_{0}$. In other words, $G_{\epsilon}$ satisfies

$$
\Delta G_{\epsilon}(x, \cdot)+\left(a_{\epsilon}-\alpha_{0}\right) G_{\epsilon}(x, \cdot)=\delta_{x}
$$

weakly in $\mathcal{D}(U)$. It is standard that $G_{\epsilon}$ exists and, since $0 \in U$, that there exists $C>0$ such that

$$
0<G_{\epsilon}(0, x) \leq C \cdot|x|^{2-n}
$$

for all $\epsilon>0$ and all $x \in \bar{U} \backslash\{0\}$. More precisely, there exists $\delta_{0}>0$ and $C_{0}>0$ such that

$$
G_{\epsilon}(0, x) \geq C_{0} \cdot|x|^{2-n} \text { and } \frac{\left|\nabla G_{\epsilon}(0, x)\right|}{|x|^{n-2}} \geq \frac{C_{0}}{|x|}
$$


for all $\epsilon>0$ and all $x \in B_{\delta_{0}}(0) \backslash\{0\}$. We let the operator

$$
L_{\epsilon}=\Delta+\left(a_{\epsilon}-\frac{u_{\epsilon}^{2^{\star}-2-p_{\epsilon}}}{|x|^{s}}\right) .
$$

We claim that there exist $\nu_{0} \in(0,1)$ and $R_{1}>0$ such that for any $\nu \in\left(0, \nu_{0}\right)$ and any $R>R_{1}$, we have that

$$
L_{\epsilon} G_{\epsilon}^{1-\nu}>0
$$

for all $x \in \Omega \backslash B_{R k_{\epsilon}}(0)$ and for all $\epsilon>0$ sufficiently small. Indeed, we let $\nu_{0} \in(0,1)$ such that for any $\nu \in\left(0, \nu_{0}\right)$, we have that

$$
\nu \cdot\left(a_{\epsilon}(x)-\alpha_{0}\right) \geq-\frac{1}{2} \alpha_{0}
$$

for all $\epsilon>0$ and all $x \in \Omega$. With (88), we get that

$$
\frac{L_{\epsilon} G_{\epsilon}^{1-\nu}}{G_{\epsilon}^{1-\nu}}(x)=\alpha_{0}+\nu \cdot\left(a_{\epsilon}(x)-\alpha_{0}\right)+\nu \cdot(1-\nu) \cdot \frac{\left|\nabla G_{\epsilon}\right|^{2}}{G_{\epsilon}^{2}}(x)-\frac{u_{\epsilon}(x)^{2^{\star}-2-p_{\epsilon}}}{|x|^{s}}
$$

for all $x \in \Omega \backslash\{0\}$ and all $\epsilon>0$. It follows from the pointwise estimate (77) that there exists $R_{1}>0$ such that for any $R>R_{1}$, we have that

$$
|x|^{2-s} u_{\epsilon}(x)^{2^{\star}-2-p_{\epsilon}} \leq \frac{1}{2} \nu(1-\nu) C_{0}^{2}
$$

for all $\epsilon>0$ and all $x \in \Omega \backslash B_{R k_{\epsilon}}(0)$. Here, $C_{0}>0$ is as in (90). We are now in position to prove (91). We let $\nu \in\left(0, \nu_{0}\right)$ and $R>R_{1}$. We first let $x \in \Omega$ such that $|x| \geq \delta_{0}$. It follows from (931) and (92) that

$$
\frac{L_{\epsilon} G_{\epsilon}^{1-\nu}}{G_{\epsilon}^{1-\nu}}(x) \geq \frac{\alpha_{0}}{2}-\frac{u_{\epsilon}(x)^{2^{\star}-2-p_{\epsilon}}}{\delta_{0}^{s}}
$$

for all $\epsilon>0$. Inequality (91) then follows with this inequality and (76). This proves (91) when $|x| \geq \delta_{0}$.

We let $x \in B_{\delta_{0}}(0) \backslash B_{R k_{\epsilon}}(0)$. It follows from (93), (90) and (94) that

$$
\frac{L_{\epsilon} G_{\epsilon}^{1-\nu}}{G_{\epsilon}^{1-\nu}}(x) \geq \frac{\alpha_{0}}{2}+\frac{\nu \cdot(1-\nu) \cdot C_{0}^{2}}{|x|^{2}}-\frac{\nu \cdot(1-\nu) \cdot C_{0}^{2}}{2 \cdot|x|^{2}}>0 .
$$

This proves (91) when $x \in B_{\delta_{0}}(0) \backslash B_{R k_{\epsilon}}(0)$. Clearly these two assertions prove inequality (91).

We let $R<R_{1}$ and $\nu \in\left(0, \nu_{0}\right)$. We claim that there exists $C(R)>0$ such that

$$
\left\{\begin{array}{ll}
L_{\epsilon}\left(C(R) \mu_{\epsilon}^{\frac{n-2}{2}-\nu(n-2)} G_{\epsilon}(0, \cdot)^{1-\nu}\right)>L_{\epsilon} u_{\epsilon} & \text { in } \Omega \backslash B_{R k_{\epsilon}}(0) \\
C(R) \mu_{\epsilon}^{\frac{n-2}{2}-\nu(n-2)} G_{\epsilon}(0, \cdot)^{1-\nu}>u_{\epsilon} & \text { on } \left.\partial \Omega \backslash B_{R k_{\epsilon}}(0)\right)
\end{array}\right\}
$$

Indeed, the first inequality is trivial since $L_{\epsilon} u_{\epsilon}=0$ and (91) holds. Concerning the second inequality, we get with the definition (27) of $\mu_{\epsilon}$, the limit (29) and (90) that

$$
\begin{aligned}
\frac{u_{\epsilon}(x)}{\mu_{\epsilon}^{\frac{n-2}{2}-\nu(n-2)} G_{\epsilon}(0, x)^{1-\nu}} \leq & C_{0}^{\nu-1} \cdot \mu_{\epsilon}^{-(n-2)(1-\nu)} \cdot|x|^{(n-2)(1-\nu)} \\
& \leq 2 \cdot C_{0}^{1-\nu} \cdot R^{(n-2)(1-\nu)}:=C(R)
\end{aligned}
$$

for all $x \in \Omega \cap \partial B_{R k_{\epsilon}}(0)$. The inequalities (95) are proved. 
Since $G_{\epsilon}(0, x)^{1-\nu}>0$ in $\overline{\Omega \cap \partial B_{R k_{\epsilon}}(0)}$ and $L_{\epsilon} G_{\epsilon}(0, x)^{1-\nu}>0$ in $\Omega \cap \partial B_{R k_{\epsilon}}(0)$, it follows from [2] that $L_{\epsilon}$ verifies the comparison principle. It then follows from (95) that

$$
u_{\epsilon}(x) \leq C(R) \mu^{\frac{n-2}{2}-\nu(n-2)} G_{\epsilon}(0, x)^{1-\nu}
$$

for all $x \in \Omega \backslash \bar{B}_{R k_{\epsilon}}(0)$. With (89), we get that there exists $C^{\prime}(R)>0$ such that

$$
u_{\epsilon}(x) \leq C^{\prime}(R) \mu_{\epsilon}^{\frac{n-2}{2}-\nu(n-2)}|x|^{2-n+\nu(n-2)}
$$

for all $x \in \Omega \backslash \bar{B}_{R k_{\epsilon}}(0)$. Up to taking $C^{\prime}(R)$ larger, it follows from (27) that this inequality holds on the whole set $\Omega$. Taking $\alpha=(n-2) \cdot(1-\nu)$, we get (86) for $\alpha$ close to $n-2$. As easily checked, this implies the inequality for all $\alpha \in(0, n-2)$. This ends the proof of (86).

Step 6.4: We are in position to prove Proposition 6.1 For all $\epsilon>0$, we let $y_{\epsilon} \in \Omega$ such that

$$
\max _{x \in \Omega}|x|^{n-2} u_{\epsilon}\left(x_{\epsilon}\right) u_{\epsilon}(x)=\left|y_{\epsilon}\right|^{n-2} u_{\epsilon}\left(x_{\epsilon}\right) u_{\epsilon}\left(y_{\epsilon}\right) .
$$

Clearly, Proposition 6.1 is equivalent to proving that

$$
\left|y_{\epsilon}\right|^{n-2} u_{\epsilon}\left(x_{\epsilon}\right) u_{\epsilon}\left(y_{\epsilon}\right)=O(1)
$$

when $\epsilon \rightarrow 0$.

Case 6.4.1: We assume that

$$
\left|y_{\epsilon}\right|=O\left(k_{\epsilon}\right)
$$

when $\epsilon \rightarrow 0$. We then get with (27) that

$$
\left|y_{\epsilon}\right|^{n-2} u_{\epsilon}\left(x_{\epsilon}\right) u_{\epsilon}\left(y_{\epsilon}\right)=O(1) \text {. }
$$

when $\epsilon \rightarrow 0$. This proves (96) in Case 6.4.1.

Case 6.4.2: We assume that

$$
\lim _{\epsilon \rightarrow 0} \frac{\left|y_{\epsilon}\right|}{k_{\epsilon}}=+\infty
$$

As in the beginning of Step 6.3 , we choose $U_{0}$ such that $\bar{\Omega} \subset \subset U_{0}$ such that $\Delta+a_{\epsilon}$ is coercive on $U_{0}$. We let $H_{\epsilon}$ be the Green's function for $\Delta+a_{\epsilon}$ on $U_{0}$ with Dirichlet boundary condition. It follows from Green's representation formula and standard estimates on the Green's function that

$$
u_{\epsilon}(x) \leq \int_{\Omega} H_{\epsilon}(x, y) \cdot \frac{u_{\epsilon}(y)^{2^{\star}-1-p_{\epsilon}}}{|y|^{s}} d y \leq C \int_{\Omega}|x-y|^{2-n} \cdot \frac{u_{\epsilon}(y)^{2^{\star}-1-p_{\epsilon}}}{|y|^{s}} d y
$$

for all $x \in \Omega$. We let

$$
\hat{v}_{\epsilon}(x)=\mu_{\epsilon}^{\frac{n-2}{2}} u_{\epsilon}\left(k_{\epsilon} x\right)
$$

for all $x \in k_{\epsilon}^{-1} \Omega$ and all $\epsilon>0$. It follows from Proposition [5.1] and (86) that for any $\alpha \in(0, n-2)$, there exists $C_{\alpha}>0$ such that

$$
\hat{v}_{\epsilon}(x) \leq \frac{C_{\alpha}}{1+|x|^{\alpha}}
$$


for all $x \in k_{\epsilon}^{-1} \Omega$ and all $\epsilon>0$. It follows from (98) and a change of variable that

$$
\begin{aligned}
\mu_{\epsilon}^{-\frac{n-2}{2}} u_{\epsilon}\left(y_{\epsilon}\right) \leq & C \int_{k_{\epsilon}^{-1} \Omega}\left|y_{\epsilon}-k_{\epsilon} y\right|^{2-n} \frac{\hat{v}_{\epsilon}(y)^{2^{\star}-1-p_{\epsilon}}}{|y|^{s}} d x \\
\leq & C \int_{k_{\epsilon}^{-1} \Omega \cap\left\{\left|y_{\epsilon}-k_{\epsilon} y\right| \geq \frac{\left|y_{\epsilon}\right|}{2}\right\}} \frac{1}{\left|y_{\epsilon}-k_{\epsilon} y\right|^{n-2}} \cdot \frac{\hat{v}_{\epsilon}(y)^{2^{\star}-1-p_{\epsilon}}}{|y|^{s}} d x \\
& +C \int_{k_{\epsilon}^{-1} \Omega \cap\left\{\left|y_{\epsilon}-k_{\epsilon} y\right|<\frac{\left|y_{\epsilon}\right|}{2}\right\}} \frac{1}{\left|y_{\epsilon}-k_{\epsilon} y\right|^{n-2}} \cdot \frac{\hat{v}_{\epsilon}(y)^{2^{\star}-1-p_{\epsilon}}}{|y|^{s}} d(\{100)
\end{aligned}
$$

We estimate the two integrals of the RHS separately. With (99), we get that

$$
\begin{aligned}
& \int_{k_{\epsilon}^{-1} \Omega \cap\left\{\left|y_{\epsilon}-k_{\epsilon} y\right| \geq \frac{\left|y_{\epsilon}\right|}{2}\right\}} \frac{1}{\left|y_{\epsilon}-k_{\epsilon} y\right|^{n-2}} \cdot \frac{\hat{v}_{\epsilon}(y)^{2^{\star}-1-p_{\epsilon}}}{|y|^{s}} d x \\
& \leq C \cdot\left|y_{\epsilon}\right|^{2-n} \int_{k_{\epsilon}^{-1} \Omega} \frac{1}{|y|^{s}\left(1+|y|^{\alpha \cdot\left(2^{\star}-1-p_{\epsilon}\right)}\right)} d y \\
& \leq C \cdot\left|y_{\epsilon}\right|^{2-n}
\end{aligned}
$$

for all $\epsilon>0$ small and $\alpha$ close enough to $n-2$. On the other hand, with (99), we get that

$$
\begin{aligned}
& \int_{k_{\epsilon}^{-1} \Omega \cap\left\{\left|y_{\epsilon}-k_{\epsilon} y\right| \leq \frac{\left|y_{\epsilon}\right|}{2}\right\}} \frac{1}{\left|y_{\epsilon}-k_{\epsilon} y\right|^{n-2}} \cdot \frac{\hat{v}_{\epsilon}(y)^{2^{\star}-1-p_{\epsilon}}}{|y|^{s}} d x \\
& \leq C \int_{k_{\epsilon}^{-1} \Omega \cap\left\{\left|y_{\epsilon}-k_{\epsilon} y\right| \leq \frac{\left|y_{\epsilon}\right|}{2}\right\}} \frac{1}{\left|y_{\epsilon}-k_{\epsilon} y\right|^{n-2}} \cdot \frac{1}{|y|^{\alpha\left(2^{\star}-1-p_{\epsilon}\right)+s}} d x \\
& \leq \frac{C \cdot k_{\epsilon}^{\alpha\left(2^{\star}-1-p_{\epsilon}\right)+s}}{\left|y_{\epsilon}\right|^{\alpha\left(2^{\star}-1-p_{\epsilon}\right)+s}} \int_{k_{\epsilon}^{-1} \Omega \cap\left\{\left|y_{\epsilon}-k_{\epsilon} y\right| \leq \frac{\left|y_{\epsilon}\right|}{2}\right\}} \frac{1}{\left|y_{\epsilon}-k_{\epsilon} y\right|^{n-2}} d y \\
& \leq \frac{C \cdot k_{\epsilon}^{\alpha\left(2^{\star}-1-p_{\epsilon}\right)+s}}{\left|y_{\epsilon}\right|^{\alpha\left(2^{\star}-1-p_{\epsilon}\right)+s}} \cdot \frac{\left|y_{\epsilon}\right|^{2}}{\left|k_{\epsilon}\right|^{n}} \\
& \leq C\left|y_{\epsilon}\right|^{2-n} \cdot\left(\frac{k_{\epsilon}}{\left|y_{\epsilon}\right|}\right)^{\left(2^{\star}-1-p_{\epsilon}\right) \alpha+s-n}
\end{aligned}
$$

Since $\lim _{\alpha \rightarrow n-2} \lim _{\epsilon \rightarrow 0}\left(2^{\star}-1-p_{\epsilon}\right) \alpha+s-n=2-s>0$, we get with (97) and $\alpha$ close enough to $n-2$ that

$$
\int_{k_{\epsilon}^{-1} \Omega \cap\left\{\left|y_{\epsilon}-k_{\epsilon} y\right| \geq \frac{\left|y_{\epsilon}\right|}{2}\right\}} \frac{1}{\left|y_{\epsilon}-k_{\epsilon} y\right|^{n-2}} \cdot \frac{\hat{v}_{\epsilon}(y)^{2^{\star}-1-p_{\epsilon}}}{|y|^{s}} d x=o\left(\left|y_{\epsilon}\right|^{2-n}\right),
$$

when $\epsilon \rightarrow 0$. Plugging together (101) and (102) into (100), we get that

$$
\mu_{\epsilon}^{-\frac{n-2}{2}} u_{\epsilon}\left(y_{\epsilon}\right)=O\left(\left|y_{\epsilon}\right|^{2-n}\right)
$$

when $\epsilon \rightarrow 0$. This proves that (96) holds in Case 6.4.2.

In both cases, we have proved that (96) holds. As easily checked, (58) and then Proposition 6.1 follow from (96) and (27). This ends Step 6.4, and therefore proves Proposition 6.1

Step 6.5: From Proposition 6.1 we can derive pointwise estimates for $v_{\epsilon}$. This is the object of the following proposition 
Proposition 6.2. Assume that the hypothesis of Proposition 6.1 are satisfied. Then there exists $C>0$ such that

$$
v_{\epsilon}(x) \leq \frac{C}{\left(1+|x|^{2}\right)^{\frac{n-2}{2}}} \text { and }\left|\nabla v_{\epsilon}\right|(x) \leq \frac{C}{\left(1+|x|^{2}\right)^{\frac{n-1}{2}}}
$$

for all $\epsilon>0$ and all $x \in \frac{U}{k_{\epsilon}} \cap\left\{x_{1}<0\right\}$, where $v_{\epsilon}$ was defined in 45) and $U$ is as in [16) with $x_{0}=0$.

Proof. The first inequality of the proposition is an immediate consequence of the estimate (58) and the definition (45) of $v_{\epsilon}$. Concerning the second inequality, we proceed by contradiction and assume that there exists a family $\left(y_{\epsilon}\right)_{\epsilon>0}$ such that $y_{\epsilon} \in U$ for all $\epsilon \rightarrow 0$ and such that

$$
\lim _{\epsilon \rightarrow 0}\left(1+\left|\frac{y_{\epsilon}}{k_{\epsilon}}\right|\right)^{n-1}\left|\nabla v_{\epsilon}\left(\frac{y_{\epsilon}}{k_{\epsilon}}\right)\right|=+\infty
$$

Case 6.5.1: we assume that $y_{\epsilon} \nrightarrow \rightarrow 0$ when $\epsilon \rightarrow 0$. It follows from the pointwise estimate (58) that for any $\delta>0$, there exists $C(\delta)>0$ such that

$$
u_{\epsilon}(x) \leq C(\delta) \mu_{\epsilon}^{\frac{n-2}{2}}
$$

for all $x \in \bar{\Omega} \backslash B_{\delta}\left(x_{0}\right)$ and all $\epsilon>0$. We then get that

$$
\Delta\left(\mu_{\epsilon}^{\frac{2-n}{2}} u_{\epsilon}\right)+a_{\epsilon} \cdot\left(\mu_{\epsilon}^{\frac{2-n}{2}} u_{\epsilon}\right)=\mu_{\epsilon}^{\frac{n-2}{2}\left(2^{\star}-2-p_{\epsilon}\right)} \frac{\left(\mu_{\epsilon}^{\frac{2-n}{2}} u_{\epsilon}\right)^{2^{\star}-1-p_{\epsilon}}}{|x|^{s}}
$$

in $\mathcal{D}^{\prime}\left(\Omega \backslash \bar{B}_{\delta}\left(x_{0}\right)\right)$. It then follows from standard elliptic theory that

$$
\left\|\mu_{\epsilon}^{\frac{2-n}{2}} u_{\epsilon}\right\|_{C^{2}\left(\bar{\Omega} \backslash B_{3 \delta}\left(x_{0}\right)\right)}=O(1)
$$

when $\epsilon \rightarrow 0$. Since $y_{\epsilon} \nrightarrow \rightarrow 0$, there exists $\delta>0$ such that, up to a subsequence, $\left|y_{\epsilon}\right| \geq 4 \delta$ for $\epsilon>0$. It follows from (104) that $\nabla u_{\epsilon}\left(\varphi\left(y_{\epsilon}\right)\right)=O\left(\mu_{\epsilon}^{\frac{n-2}{2}}\right)$ when $\epsilon \rightarrow 0$. A contradiction with (103). This proves the Proposition in Case 6.5.1.

Case 6.5.2: We assume that

$$
\lim _{\epsilon \rightarrow 0} y_{\epsilon}=0 \text { and } \lim _{\epsilon \rightarrow 0} \frac{\left|y_{\epsilon}\right|}{k_{\epsilon}}=+\infty .
$$

We let $\varphi$ as in (16) with $x_{0}=0$ and define

$$
h_{\epsilon}(x):=\frac{\left|y_{\epsilon}\right|^{n-2}}{k_{\epsilon}^{\frac{n-2}{2}}} u_{\epsilon} \circ \varphi\left(\left|y_{\epsilon}\right| x\right)
$$

for all $x \in \frac{U}{\left|y_{\epsilon}\right|} \cap\left\{x_{1} \leq 0\right\}$. It follows from (58) and (29) that there exists $C>0$ such that

$$
h_{\epsilon}(x) \leq C \cdot|x|^{2-n}
$$

for all $x \in \frac{U}{\left|y_{\epsilon}\right|} \cap\left\{x_{1} \leq 0\right\}, x \neq 0$. We let

$$
\Delta_{\bar{g}_{\epsilon}}=\bar{g}_{\epsilon}^{i j}\left(\partial_{i j}-\Gamma_{i j}^{k}\left(\bar{g}_{\epsilon}\right) \partial_{k}\right),
$$

the Laplace-Beltrami operator for the metric $\left(\bar{g}_{\epsilon}\right)_{i j}=\left(\partial_{i} \varphi, \partial_{j} \varphi\right)\left(k_{\epsilon} x\right)$. In this expression, the $\bar{g}_{\epsilon}^{i j}=\left(\bar{g}_{\epsilon}^{-1}\right)_{i j}$ are the coordinates of the inverse of the tensor $\bar{g}_{\epsilon}$ and 
the $\Gamma_{i j}^{k}\left(\bar{g}_{\epsilon}\right)$ are the Christoffel symbols associated to the metric $\bar{g}_{\epsilon}$. After a change of variables, (23) rewrites as

$$
\Delta_{\bar{g}_{\epsilon}} h_{\epsilon}+\left|y_{\epsilon}\right|^{2} a_{\epsilon}\left(\varphi\left(\left|y_{\epsilon}\right| x\right)\right) h_{\epsilon}=k_{\epsilon}^{p_{\epsilon} \frac{n-2}{2}}\left(\frac{k_{\epsilon}}{\left|y_{\epsilon}\right|}\right)^{2-s-p_{\epsilon}(n-2)} \frac{h_{\epsilon}^{2^{\star}-1-p_{\epsilon}}}{\left|\frac{\varphi\left(\left|y_{\epsilon}\right| x\right)}{\left|y_{\epsilon}\right|}\right|^{s}}
$$

in $\mathcal{D}^{\prime}\left(\frac{U}{\left|y_{\epsilon}\right|} \cap\left\{x_{1}<0\right\}\right)$. Since (29), (105) and (106) hold and since $s \in(0,2)$, there exists $p>\frac{n}{2}$ such that

$$
\Delta_{\bar{g}_{\epsilon}} h_{\epsilon}+\left|y_{\epsilon}\right|^{2} a_{\epsilon}\left(\varphi\left(\left|y_{\epsilon}\right| x\right)\right) h_{\epsilon}=f_{\epsilon} \text { in } \mathcal{D}^{\prime}\left(\frac{U}{\left|y_{\epsilon}\right|} \cap\left\{x_{1}<0\right\}\right),
$$

where $f_{\epsilon} \in L_{l o c}^{p}\left(\frac{U}{\left|y_{\epsilon}\right|} \cap\left\{x_{1} \leq 0\right\} \backslash\{0\}\right)$ uniformly wrt $\epsilon \rightarrow 0$. Since $h_{\epsilon} \equiv 0$ on $\frac{U}{\left|y_{\epsilon}\right|} \cap\left\{x_{1}=0\right\}$ and (106) holds, it follows from standard elliptic theory that there exists for any $\delta_{1}>\delta_{2}>0$, there exists $C^{\prime}\left(\delta_{1}, \delta_{2}\right)>0$ such that

$$
\left\|h_{\epsilon}\right\|_{C^{1}\left(\left(B_{\delta_{1}}(0) \backslash B_{\delta_{2}}(0)\right) \cap\left\{x_{1} \leq 0\right\}\right)} \leq C^{\prime}\left(\delta_{1}, \delta_{2}\right)
$$

for all $\epsilon>0$. It then follows that

$$
\left|\nabla h_{\epsilon}\left(\frac{y_{\epsilon}}{\left|y_{\epsilon}\right|}\right)\right|=O(1)
$$

when $\epsilon \rightarrow 0$. Coming back to the definitions of $h_{\epsilon}$ and $v_{\epsilon}$, we get a contradiction with (103). This proves the Proposition in Case 6.5.2.

Case 6.5.3: We assume that

$$
\left|y_{\epsilon}\right|=O\left(k_{\epsilon}\right)
$$

when $\epsilon \rightarrow 0$. In this case, It follows from Proposition 5.1 that $\left|\nabla v_{\epsilon}\left(\frac{y_{\epsilon}}{k_{\epsilon}}\right)\right|=O(1)$ when $\epsilon \rightarrow 0$. We get a contradiction with (103). This proves the Proposition in Case 3 .

In all the cases, we have contradicted (103). This proves Proposition 6.2

Corollary 6.1. Let $\left(u_{\epsilon}\right)_{\epsilon>0}$ as in the hypothesis of Proposition 6.1. Then there exists $H \in C^{1}(\bar{\Omega} \backslash\{0\})$ such that

$$
u_{\epsilon}\left(x_{\epsilon}\right) u_{\epsilon} \rightarrow H \text { in } C_{l o c}^{1}(\bar{\Omega} \backslash\{0\})
$$

when $\epsilon \rightarrow 0$.

Proof. We let $H_{\epsilon}(x):=u_{\epsilon}\left(x_{\epsilon}\right) u_{\epsilon}(x)$ for all $x \in \Omega$ and all $\epsilon>0$. It follows from Proposition 6.1 that for any open subset $U$ such that $\bar{U} \subset \bar{\Omega} \backslash\{0\}$, there exists $C(U)>0$ such that $\left|H_{\epsilon}(x)\right| \leq C(U)$ for all $x \in U$ and all $\epsilon>0$. Equation (23) rewrites as

$$
\Delta H_{\epsilon}+a_{\epsilon} H_{\epsilon}=u_{\epsilon}\left(x_{\epsilon}\right)^{2+p_{\epsilon}-2^{\star}} \frac{H_{\epsilon}^{2^{\star}-1-p_{\epsilon}}}{|x|^{s}}
$$

in $\Omega$. The conclusion of the Corollary is then a consequence of standard elliptic theory. 


\section{Pohozaev identity And PRoOF of The TheOREMS}

In this section, we prove the following estimate:

Proposition 7.1. Let $\Omega$ be a smooth bounded domain of $\mathbb{R}^{n}, n \geq 4$. We let $s \in(0,2)$. We let $\left(p_{\epsilon}\right)_{\epsilon>0}$ such that $p_{\epsilon} \in\left[0,2^{\star}-2\right)$ for all $\epsilon>0$ and (22) holds. We consider $\left(u_{\epsilon}\right)_{\epsilon>0} \in H_{1,0}^{2}(\Omega)$ such that (14), (15), (23), 24) and (26) hold. We let $\mu_{\epsilon}$ as in (27) and $v$ as in Proposition 5.1. Then, we have that

$$
\lim _{\epsilon \rightarrow 0} \frac{p_{\epsilon}}{\mu_{\epsilon}}=\frac{(n-s) \int_{\partial \mathbb{R}_{-}^{n}}|x|^{2}|\nabla v|^{2} d x}{n(n-2)^{2} \mu_{s}\left(\mathbb{R}_{-}^{n}\right)^{\frac{n-s}{2-s}}} \cdot H(0) .
$$

In this expression, $H(0)$ is the mean curvature of the oriented boundary $\partial \Omega$ at 0 .

We prove the Proposition in the sequel, and postpone the proofs of Theorems 1.1 and 1.4 to the end of the section. We let $p_{\epsilon} \geq 0$ such that $\lim _{\epsilon \rightarrow 0} p_{\epsilon}=0$. We let $u_{\epsilon}, a_{\epsilon}$ and $a$ as in (14), (15), (23), (24) and (26). We assume that $0<s<2$ and let $x_{\epsilon}, \mu_{\epsilon}, k_{\epsilon}$ as in (27). Since $\lim _{\epsilon \rightarrow 0} x_{\epsilon}=0$, we consider the chart $\varphi$ defined in (16) with $x_{0}=0$.

Step 7.1: We provide a Pohozaev-type identity for $u_{\epsilon}$. It follows from Proposition 8.1 that $u_{\epsilon} \in C^{1}(\bar{\Omega})$ and that $\Delta u_{\epsilon} \in L^{p}(\Omega)$ for all $p \in\left(1, \frac{n}{s}\right)$. In the sequel, we denote by $\nu(x)$ the outward normal vector at $x \in \partial \Omega$ of the oriented hypersurface $\partial \Omega$ (oriented as the boundary of $\Omega$ ). Integrating by parts, we get that

$$
\begin{aligned}
& \int_{\Omega} x^{i} \partial_{i} u_{\epsilon} \Delta u_{\epsilon} d x \\
& =-\int_{\partial \Omega} x^{i} \partial_{i} u_{\epsilon} \partial_{\nu} u_{\epsilon} d \sigma+\int_{\Omega} \partial_{j}\left(x^{i} \partial_{i} u_{\epsilon}\right) \partial_{j} u_{\epsilon} d x \\
& =-\int_{\partial \Omega} x^{i} \partial_{i} u_{\epsilon} \partial_{\nu} u_{\epsilon} d \sigma+\int_{\Omega}\left|\nabla u_{\epsilon}\right|^{2} d x+\int_{\Omega} x^{i} \partial_{i} \frac{\left|\nabla u_{\epsilon}\right|^{2}}{2} d x \\
& =\left(1-\frac{n}{2}\right) \int_{\Omega}\left|\nabla u_{\epsilon}\right|^{2} d x+\int_{\partial \Omega}\left((x, \nu) \frac{\left|\nabla u_{\epsilon}\right|^{2}}{2}-x^{i} \partial_{i} u_{\epsilon} \partial_{\nu} u_{\epsilon}\right) d \sigma \\
& =\left(1-\frac{n}{2}\right)\left(\int_{\partial \Omega} u_{\epsilon} \partial_{\nu} u_{\epsilon} d \sigma+\int_{\Omega} u_{\epsilon} \Delta u_{\epsilon} d x\right) \\
& +\int_{\partial \Omega}\left((x, \nu) \frac{\left|\nabla u_{\epsilon}\right|^{2}}{2}-x^{i} \partial_{i} u_{\epsilon} \partial_{\nu} u_{\epsilon}\right) d \sigma .
\end{aligned}
$$

Using the equation (23) in the RHS, we get that

$$
\begin{aligned}
& \int_{\Omega} x^{i} \partial_{i} u_{\epsilon} \Delta u_{\epsilon} d x=\left(1-\frac{n}{2}\right)\left(\int_{\Omega} \frac{u_{\epsilon}^{2^{\star}-p_{\epsilon}}}{|x|^{s}} d x-\int_{\Omega} a_{\epsilon} u_{\epsilon}^{2} d x\right) \\
& +\int_{\partial \Omega}\left(\left(1-\frac{n}{2}\right) u_{\epsilon} \partial_{\nu} u_{\epsilon}+(x, \nu) \frac{\left|\nabla u_{\epsilon}\right|^{2}}{2}-x^{i} \partial_{i} u_{\epsilon} \partial_{\nu} u_{\epsilon}\right) d \sigma .
\end{aligned}
$$


On the other hand, using the equation (23) satisfied by $u_{\epsilon}$, we get that

$$
\begin{aligned}
& \int_{\Omega} x^{i} \partial_{i} u_{\epsilon} \Delta u_{\epsilon} d x=\int_{\Omega} x^{i} \partial_{i} u_{\epsilon} \frac{u_{\epsilon}^{2^{\star}-1-\epsilon}}{|x|^{s}} d x-\int_{\Omega} x^{i} \partial_{i} u_{\epsilon} a_{\epsilon} u_{\epsilon} d x \\
& =\int_{\Omega} x^{i}|x|^{-s} \partial_{i}\left(\frac{u_{\epsilon}^{2^{\star}-p_{\epsilon}}}{2^{\star}-p_{\epsilon}}\right) d x-\int_{\Omega} x^{i} \partial_{i} u_{\epsilon} a_{\epsilon} u_{\epsilon} d x \\
& =-\int_{\Omega} \partial_{i}\left(x^{i}|x|^{-s}\right) \frac{u_{\epsilon}^{2^{\star}-p_{\epsilon}}}{2^{\star}-p_{\epsilon}} d x+\int_{\partial \Omega} \frac{(x, \nu)}{2^{\star}-p_{\epsilon}} \cdot \frac{u_{\epsilon}^{2^{\star}-p_{\epsilon}}}{|x|^{s}} d \sigma-\int_{\Omega} x^{i} \partial_{i} u_{\epsilon} a_{\epsilon} u_{\epsilon} d x \\
& =-\int_{\Omega} \frac{n-s}{|x|^{s}} \cdot \frac{u_{\epsilon}^{2^{\star}-p_{\epsilon}}}{2^{\star}-p_{\epsilon}} d x+\frac{1}{2} \int_{\Omega}\left(n a_{\epsilon}+x^{i} \partial_{i} a_{\epsilon}\right) u_{\epsilon}^{2} d x \\
& +\int_{\partial \Omega} \frac{(x, \nu)}{2^{\star}-p_{\epsilon}} \cdot \frac{u_{\epsilon}^{2^{\star}-p_{\epsilon}}}{|x|^{s}} d \sigma-\int_{\partial \Omega} \frac{(x, \nu)}{2} a_{\epsilon} u_{\epsilon}^{2} d \sigma .
\end{aligned}
$$

Plugging together (107) and (108), we get that

$$
\begin{aligned}
& \left(\frac{n-2}{2}-\frac{n-s}{2^{\star}-p_{\epsilon}}\right) \int_{\Omega} \frac{u_{\epsilon}^{2^{\star}-p_{\epsilon}}}{|x|^{s}} d x+\int_{\Omega}\left(a_{\epsilon}+\frac{\left(x, \nabla a_{\epsilon}\right)}{2}\right) u_{\epsilon}^{2} d x \\
& =\int_{\partial \Omega}\left(-\frac{n-2}{2} u_{\epsilon} \partial_{\nu} u_{\epsilon}+(x, \nu) \frac{\left|\nabla u_{\epsilon}\right|^{2}}{2}\right. \\
& \left.-x^{i} \partial_{i} u_{\epsilon} \partial_{\nu} u_{\epsilon}-\frac{(x, \nu)}{2^{\star}-p_{\epsilon}} \cdot \frac{u_{\epsilon}^{2^{\star}-p_{\epsilon}}}{|x|^{s}}\right) d \sigma+\int_{\partial \Omega} \frac{(x, \nu)}{2} a_{\epsilon} u_{\epsilon}^{2} d x
\end{aligned}
$$

for all $\epsilon>0$. Since $u_{\epsilon} \equiv 0$ on $\partial \Omega$, we get that

$$
\begin{aligned}
& \frac{(n-2) p_{\epsilon}}{2 \cdot\left(2^{\star}-p_{\epsilon}\right)} \int_{\Omega} \frac{u_{\epsilon}^{2^{\star}-p_{\epsilon}}}{|x|^{s}} d x-\int_{\Omega}\left(a_{\epsilon}+\frac{\left(x, \nabla a_{\epsilon}\right)}{2}\right) u_{\epsilon}^{2} d x \\
& =\frac{1}{2} \int_{\partial \Omega}(x, \nu)\left|\nabla u_{\epsilon}\right|^{2} d \sigma .
\end{aligned}
$$

Step 7.2: We first deal with the RHS of (110). We take $\varphi$ as in (16) with $x_{0}=0$. With the pointwise limit of Corollary [6.1] we get that

$$
\int_{\partial \Omega}(x, \nu)\left|\nabla u_{\epsilon}\right|^{2} d \sigma=\int_{\partial \Omega \cap \varphi(U)}(x, \nu)\left|\nabla u_{\epsilon}\right|^{2} d \sigma+o\left(\mu_{\epsilon}\right)
$$

when $\epsilon \rightarrow 0$ as soon as $n \geq 4$. With a change of variable, we get that

$$
\begin{aligned}
& \int_{\partial \Omega}(x, \nu)\left|\nabla u_{\epsilon}\right|^{2} d \sigma= \\
& (1+o(1)) \cdot \int_{D_{\epsilon}}\left(\frac{\varphi\left(k_{\epsilon} x\right)}{k_{\epsilon}}, \nu \circ \varphi\left(k_{\epsilon} x\right)\right)\left|\nabla v_{\epsilon}\right|_{\tilde{g}_{\epsilon}}^{2} \sqrt{\left|\tilde{g}_{\epsilon}\right|} d x \\
& +o\left(\mu_{\epsilon}^{n-2}\right)
\end{aligned}
$$

where the metric $\tilde{g}_{\epsilon}$ is such that $\left(\tilde{g}_{\epsilon}\right)_{i j}=\left(\partial_{i} \varphi, \partial_{j} \varphi\right)\left(k_{\epsilon} x\right)$ for all $i, j=2, \ldots, n$, $\left|\tilde{g}_{\epsilon}\right|=\operatorname{det}\left(\tilde{g}_{\epsilon}\right)$ and

$$
D_{\epsilon}=\frac{U}{k_{\epsilon}} \cap\left\{x_{1}=0\right\} .
$$

Using the expression of $\varphi$ (see (16)), we get that

$$
\nu(\varphi(x))=\frac{\left(1,-\partial_{2} \varphi_{0}(x), \ldots,-\partial_{n} \varphi_{0}(x)\right)}{\sqrt{1+\sum_{i=2}^{n}\left(\partial_{i} \varphi_{0}(x)\right)^{2}}}
$$


for all $x \in U \cap\left\{x_{1}=0\right\}$. We then get that

$$
(\nu \circ \varphi(x), \vec{X})=\left(1+O\left(|x|^{2}\right)\right) \cdot\left(X^{1}-\sum_{i=2}^{n} X^{i} \partial_{i} \varphi_{0}(x)\right)
$$

for all $x \in U \cap\left\{x_{1}=0\right\}$ and all $\vec{X} \in \mathbb{R}^{n}$. In this expression $O(1)$ is bounded for $x \in U \cap\left\{x_{1}=0\right\}$ and $\vec{X} \in \mathbb{R}^{n}$. With the expression of $\varphi$ (see (16)), we get that

$$
\begin{aligned}
& \left(\varphi\left(k_{\epsilon} x\right), \nu \circ \varphi\left(k_{\epsilon} x\right)\right) \\
& =\left(1+O\left(k_{\epsilon}^{2}|x|^{2}\right)\right)\left(\varphi_{0}\left(k_{\epsilon} x\right)-k_{\epsilon} \sum_{i=2}^{n} x^{i} \partial_{i} \varphi_{0}\left(k_{\epsilon} x\right)\right) \\
& =\left(1+O\left(k_{\epsilon}^{2}|x|^{2}\right)\right) \cdot\left(-\frac{1}{2} k_{\epsilon}^{2} \partial_{i j} \varphi(0) x^{i} x^{j}+O(1)\left(k_{\epsilon}^{3}|x|^{3}\right)\right)
\end{aligned}
$$

for $\epsilon>0$ and $x \in \frac{U}{k_{\epsilon}} \cap\left\{x_{1}=0\right\}$. Plugging (112) into (111), using the estimates of Proposition 6.1 Lebesgue's convergence theorem and letting $\epsilon \rightarrow 0$, we get that

$$
\int_{\partial \Omega}(x, \nu)\left|\nabla u_{\epsilon}\right|^{2} d \sigma=\left(-\frac{1}{2} \int_{\partial \mathbb{R}_{-}^{n}} \partial_{i j} \varphi_{0}(0) x^{i} x^{j}|\nabla v|^{2} d x+o(1)\right) \cdot k_{\epsilon}
$$

when $n \geq 4$ and where $\lim _{\epsilon \rightarrow 0} o(1)=0$.

Step 7.3: It follows from Proposition 6.1

$$
\int_{\Omega} u_{\epsilon}^{2} d x=o\left(\mu_{\epsilon}\right)
$$

when $\epsilon \rightarrow 0$ and as soon as $n \geq 4$. Plugging (113) into (110), using (24) and (114), we get that

$$
\begin{aligned}
& \left(\frac{n-2}{2 \cdot 2^{\star}} \mu_{s}\left(\mathbb{R}_{-}^{n}\right)^{\frac{n-s}{2-s}}+o(1)\right) p_{\epsilon} \\
& =\left(-\frac{1}{4} \int_{\partial \mathbb{R}_{-}^{n}} \partial_{i j} \varphi_{0}(0) x^{i} x^{j}|\nabla v|^{2} d x+o(1)\right) \cdot \mu_{\epsilon}
\end{aligned}
$$

where $\lim _{\epsilon \rightarrow 0} o(1)=0$ and when $n \geq 4$. With (115), we get that

$$
\begin{aligned}
& \lim _{\epsilon \rightarrow 0} \frac{n-2}{2 \cdot 2^{\star}} \mu_{s}\left(\mathbb{R}_{-}^{n}\right)^{\frac{n-s}{2-s}} \cdot \frac{p_{\epsilon}}{\mu_{\epsilon}} \\
& =-\frac{1}{4} \int_{\partial \mathbb{R}_{-}^{n}} \partial_{i j} \varphi_{0}(0) x^{i} x^{j}|\nabla v|^{2} d x
\end{aligned}
$$

when $n \geq 4$. We consider the second fondamental form associated to $\partial \Omega$, namely

$$
I I_{p}(x, y)=\left(d \nu_{p} x, y\right)
$$

for all $p \in \partial \Omega$ and all $x, y \in T_{p} \partial \Omega$ (recall that $\nu$ is the outward normal vector at the hypersurface $\partial \Omega$ ). In the canonical basis of $\partial \mathbb{R}_{-}^{n}=T_{0} \partial \Omega$, the matrix of the bilinear form $I I_{0}$ is $-D_{0}^{2} \varphi_{0}$, where $D_{0}^{2} \varphi_{0}$ is the Hessian matrix of $\varphi_{0}$ at 0 . With this remark and (115), we get that

$$
\lim _{\epsilon \rightarrow 0} \frac{p_{\epsilon}}{\mu_{\epsilon}}=\frac{(n-s)}{(n-2)^{2}} \mu_{s}\left(\mathbb{R}_{-}^{n}\right)^{-\frac{n-s}{2-s}} \cdot \int_{\partial \mathbb{R}_{-}^{n}} I I_{0}(x, x)|\nabla v|^{2} d x
$$


when $n \geq 4$. Since $v \geq 0$, that $v \in C^{2}\left(\mathbb{R}_{-}^{n}\right)$ and $v$ verifies (52), it follows from the strong maximum principle that $v>0$ in $\mathbb{R}_{-}^{n}$. Moreover, it follows from the definition (45) and the pointwise estimate (58) that there exists $C>0$ such that

$$
v(x) \leq \frac{C}{\left(1+|x|^{2}\right)^{\frac{n-2}{2}}}
$$

for all $x \in \mathbb{R}_{-}^{n}$. We let $\tilde{v}(x):=|x|^{2-n} v\left(\frac{x}{|x|^{2}}\right)$ be the Kelvin transform of $v$. As easily checked, $\tilde{v} \in C^{2}\left(\overline{\mathbb{R}_{-}^{n}} \backslash\{0\}\right)$ and verifies

$$
\Delta \tilde{v}=\frac{\tilde{v}^{2^{\star}-1}}{|x|^{s}} \text { in } \mathbb{R}_{-}^{n} \text { and } \tilde{v}(x) \leq \frac{C}{\left(1+|x|^{2}\right)^{\frac{n-2}{2}}}
$$

for all $x \in \mathbb{R}_{-}^{n}$. Since $\tilde{v}$ vanishes on $\partial \mathbb{R}_{-}^{n}$, it then follows from standard elliptic theory that $\tilde{v} \in C^{1}\left(\overline{\mathbb{R}_{-}^{n}}\right)$ and then, that there exists $C>0$ such that $\tilde{v}(x) \leq C|x|$ for all $x \in B_{1}(0) \cap \mathbb{R}_{-}^{n}$. Coming back to the function $v$, we get that tehre exists $C>0$ such that

$$
v(x) \leq \frac{C}{\left(1+|x|^{2}\right)^{\frac{n-1}{2}}}
$$

for all $x \in \mathbb{R}_{-}^{n}$. It follows from Proposition 1.2 of Appendix B that there exists $w \in C^{2}\left(\mathbb{R}_{-}^{\star} \times \mathbb{R}\right)$ such that $v\left(x_{1}, x^{\prime}\right)=w\left(x_{1},\left|x^{\prime}\right|\right)$ for all $\left(x_{1}, x^{\prime}\right) \in \mathbb{R}_{-}^{\star} \times \mathbb{R}^{n-1}$. In particular, $|\nabla v|\left(0, x^{\prime}\right)$ is radially symmetrical wrt $x^{\prime} \in \partial \mathbb{R}_{-}^{n}$. Since we have chosen a chart $\varphi$ that is Euclidean at 0 , we get that

$$
\begin{aligned}
\int_{\partial \mathbb{R}_{-}^{n}} I I_{0}(x, x)|\nabla v|^{2} d x & =\frac{\sum_{i=2}^{n}\left(I I_{0}\right)^{i i}}{n} \int_{\partial \mathbb{R}_{-}^{n}}|x|^{2}|\nabla v|^{2} d x \\
& =\frac{H(0)}{n} \int_{\partial \mathbb{R}_{-}^{n}}|x|^{2}|\nabla v|^{2} d x
\end{aligned}
$$

Note that we have used here that in the chart $\varphi$ defined in (16), the matrix of the first fundamental form at 0 is the identity. Plugging thsi last inequality in (116), we get that

$$
\lim _{\epsilon \rightarrow 0} \frac{p_{\epsilon}}{\mu_{\epsilon}}=\frac{(n-s) \int_{\partial \mathbb{R}_{-}^{n}}|x|^{2}|\nabla v|^{2} d x}{n(n-2)^{2} \mu_{s}\left(\mathbb{R}_{-}^{n}\right)^{\frac{n-s}{2-s}}} \cdot H(0)
$$

when $n \geq 4$.

Step 7.4: We are now in position to prove Theorems 1.1 and 1.4 We prove Theorem 1.1 by contradiction and assume that there are no extremals for (2). It follows from Propositions 4.1 and 4.2 that there exists $u_{\epsilon} \in H_{1,0}^{2}(\Omega)$ such that (23), (24) and (26) hold with $a_{\epsilon} \equiv 0$ and $p_{\epsilon}=\epsilon$. Since $0<s<2$, then (117) holds with $p_{\epsilon}=\epsilon$ when $n \geq 4$. We then get that $H(0) \geq 0$. A contradiction with the assumptions of Theorem 1.1. This proves the first point of Theorem 1.1] when $n \geq 4$. Concerning the compactness, any sequence of minimizers of (2) satisfies (23) and (24) with $p_{\epsilon} \equiv 0$ and $a \equiv 0$. If the sequence of minimizers blows up, we get with (117) that $H(0)=0$. A contradiction with our initial assumption. Then we get that the sequence does not blow up. It then follows from standard elliptic theory that it converges in $H_{1,0}^{2}(\Omega)$. This proves Theorem 1.1 when $n \geq 4$. 
Concerning Theorem 1.4 the proof is quite similar to that of Theorem 1.1 We assume that the conclusion of the theorem does not hold. It follows from Propositions 4.1 and 4.2 that there exists $u_{\epsilon} \in H_{1,0}^{2}(\Omega)$ such that (23), (24) and (26) hold with $a_{\epsilon} \equiv a$ and $p_{\epsilon}=\epsilon$. The proof is then the same as the proof of Theorem 1.1

\section{Appendix: Regularity of Weak solutions}

In this appendix, we prove the following regularity result:

Proposition 8.1. Let $\Omega$ be a smooth bounded domain of $\mathbb{R}^{n}, n \geq 3$. We let $s \in(0,2)$ and $a \in C^{0}(\bar{\Omega})$. We let $\epsilon \in\left[0,2^{\star}-2\right)$ and consider $u \in H_{1,0}^{2}(\Omega)$ a weak solution of

$$
\Delta u+a u=\frac{|u|^{2^{\star}-2-\epsilon} u}{|x|^{s}} \text { in } \mathcal{D}^{\prime}(\Omega)
$$

Then there exists $\theta \in(0,1)$ such that $u \in C^{1, \theta}(\bar{\Omega})$.

Proof. Step 8.1: We follow the strategy developed by Trudinger. Let $\beta \geq 1$, and $L>0$. We let

$$
G_{L}(t)= \begin{cases}|t|^{\beta-1} t & \text { if }|t| \leq L \\ \beta L^{\beta-1}(t-L)+L^{\beta} & \text { if } t \geq L \\ \beta L^{\beta-1}(t+L)-L^{\beta} & \text { if } t \leq-L\end{cases}
$$

and

$$
H_{L}(t)= \begin{cases}|t|^{\frac{\beta-1}{2}} t & \text { if }|t| \leq L \\ \frac{\beta+1}{2} L^{\frac{\beta-1}{2}}(t-L)+L^{\frac{\beta+1}{2}} & \text { if } t \geq L \\ \frac{\beta+1}{2} L^{\frac{\beta-1}{2}}(t+L)-L^{\frac{\beta+1}{2}} & \text { if } t \leq-L\end{cases}
$$

As easily checked,

$$
0 \leq t G_{L}(t) \leq H_{L}(t)^{2} \text { and } G_{L}^{\prime}(t)=\frac{4 \beta}{(\beta+1)^{2}}\left(H_{L}^{\prime}(t)\right)^{2}
$$

for all $t \in \mathbb{R}$ and all $L>0$. Let $\eta \in C_{c}^{\infty}\left(\mathbb{R}^{n}\right)$. As easily checked, $\eta^{2} G_{L}(u), \eta H_{L}(u) \in$ $H_{1,0}^{2}(\Omega)$. With the equation verified by $u$, we get that

$$
\int_{\Omega} \nabla u \nabla\left(\eta^{2} G_{L}(u)\right) d x=\int_{\Omega} \frac{|u|^{2^{\star}-2-\epsilon}}{|x|^{s}} \eta^{2} u G_{L}(u) d x-\int_{\Omega} a \eta^{2} u G_{L}(u) d x .
$$

We let $J_{L}(t)=\int_{0}^{t} G_{L}(\tau) d \tau$ for all $t \in \mathbb{R}$. Integrating by parts, we get that

$$
\begin{aligned}
& \int_{\Omega} \nabla u \nabla\left(\eta^{2} G_{L}(u)\right) d x=\int_{\Omega} \eta^{2} G_{L}^{\prime}(u)|\nabla u|^{2} d x+\int_{\Omega} \nabla \eta^{2} \nabla J_{L}(u) d x \\
& =\frac{4 \beta}{(\beta+1)^{2}} \int_{\Omega} \eta^{2}\left|\nabla H_{L}(u)\right|^{2} d x+\int_{\Omega}\left(\Delta \eta^{2}\right) J_{L}(u) d x \\
& =\frac{4 \beta}{(\beta+1)^{2}} \int_{\Omega}\left|\nabla\left(\eta H_{L}(u)\right)\right|^{2} d x+\frac{4 \beta}{(\beta+1)^{2}} \int_{\Omega} \eta \Delta \eta\left|H_{L}(u)\right|^{2} d x \\
& +\int_{\Omega}\left(\Delta \eta^{2}\right) J_{L}(u) d x
\end{aligned}
$$


On the other hand, with Hölder's inequality and the definition of $\mu_{s}\left(\mathbb{R}^{n}\right)$, we then get that

$$
\begin{aligned}
& \int_{\Omega}\left(\frac{|u|^{2^{\star}-2-\epsilon}}{|x|^{s}}-a\right) \cdot \eta^{2} u G_{L}(u) d x \leq \int_{\Omega}\left(|a|+\frac{|u|^{2^{\star}-2-\epsilon}}{|x|^{s}}\right) \cdot\left(\eta H_{L}(u)\right)^{2} d x \\
& \leq\left(\int_{\Omega \cap \operatorname{Supp} \eta} \frac{\left(|a| \cdot|x|^{s}+|u|^{2^{\star}-2-p_{\epsilon}}\right)^{\frac{2^{\star}-\epsilon}{2^{\star}-2-\epsilon}}}{|x|^{s}}\right)^{1-\frac{2}{2^{\star}-\epsilon}} \\
& \times\left(\int_{\Omega} \frac{\left|\eta H_{L}(u)\right|^{2^{\star}}}{|x|^{s}}\right)^{\frac{2}{2^{\star}}} \times\left(\int_{\Omega} \frac{d x}{|x|^{s}}\right)^{\frac{2 \epsilon}{2^{\star} \cdot\left(2^{\star}-\epsilon\right)}} \\
& \leq \alpha \cdot \int_{\Omega}\left|\nabla\left(\eta H_{L}(u)\right)\right|^{2} d x
\end{aligned}
$$

where

$$
\begin{aligned}
\alpha:= & \left(\int_{\Omega \cap \operatorname{Supp} \eta} \frac{\left(|a| \cdot|x|^{s}+|u|^{2^{\star}-2-p_{\epsilon}}\right)^{\frac{2^{\star}-\epsilon}{2^{\star}-2-\epsilon}}}{|x|^{s}} d x\right)^{1-\frac{2}{2^{\star}-\epsilon}} \\
& \times \mu_{s}\left(\mathbb{R}^{n}\right)^{-1}\left(\int_{\Omega} \frac{d x}{|x|^{s}}\right)^{\frac{2 \epsilon}{2^{\star} \cdot\left(2^{\star}-\epsilon\right)}}
\end{aligned}
$$

Plugging (119) and (120) into (118), we get that

$$
A \cdot \int_{\Omega}\left|\nabla\left(\eta H_{L}(u)\right)\right|^{2} d x \leq \frac{4 \beta}{(\beta+1)^{2}} \int_{\Omega}|\eta \Delta \eta|\left|H_{L}(u)\right|^{2} d x+\int_{\Omega}\left|\Delta\left(\eta^{2}\right) J_{L}(u)\right| d x
$$

where

$$
\begin{aligned}
A:= & \frac{4 \beta}{(\beta+1)^{2}}-\left(\int_{\Omega \cap \operatorname{Supp} \eta} \frac{\left(|a| \cdot|x|^{s}+|u|^{2^{\star}-2-p_{\epsilon}}\right)^{\frac{2^{\star}-\epsilon}{2^{\star}-2-\epsilon}}}{|x|^{s}} d x\right)^{1-\frac{2}{2^{\star}-\epsilon}} \\
& \times \mu_{s}\left(\mathbb{R}^{n}\right)^{-1}\left(\int_{\Omega} \frac{d x}{|x|^{s}}\right)^{\frac{2 \epsilon}{2^{\star} \cdot\left(2^{\star}-\epsilon\right)}}
\end{aligned}
$$

Step 8.2: We let $p_{0}=\sup \left\{p \geq 1 / u \in L^{p}(\Omega)\right\}$. It follows from Sobolev's embedding theorem that $p_{0} \geq \frac{2 n}{n-2}$. We claim that

$$
p_{0}=+\infty
$$

We proceed by contradiction and assume that

$$
p_{0}<\infty
$$

Let $p \in\left(2, p_{0}\right)$. It follows from the definition of $p_{0}$ that $u \in L^{p}(\Omega)$. Let $\beta=p-1>$ 1 . For any $x \in \bar{\Omega}$, we let $\delta_{x}>0$ such that

$$
\begin{aligned}
& \left(\int_{\Omega \cap B_{2 \delta_{x}}(x)} \frac{\left(|a| \cdot|x|^{s}+|u|^{2^{\star}-2-p_{\epsilon}}\right)^{\frac{2^{\star}-\epsilon}{2^{\star}-2-\epsilon}}}{|x|^{s}} d x\right)^{1-\frac{2}{2^{\star}-\epsilon}} \mu_{s}\left(\mathbb{R}^{n}\right)^{-1} \\
& \times\left(\int_{\Omega} \frac{d x}{|x|^{s}}\right)^{\frac{2 \epsilon}{2^{\star} \cdot\left(2^{\star}-\epsilon\right)}} \leq \frac{2 \beta}{(\beta+1)^{2}}
\end{aligned}
$$


Since $\bar{\Omega}$ is compact, we get that there exists $x_{1}, \ldots, x_{N} \in \bar{\Omega}$ such that

$$
\bar{\Omega} \subset \bigcup_{i=1}^{N} B_{\delta_{x_{i}}}\left(x_{i}\right) .
$$

We fix $i \in\{1, \ldots, N\}$ and let $\eta \in C^{\infty}\left(B_{2 \delta_{x_{i}}}\left(x_{i}\right)\right)$ such that $\eta(x)=1$ for all $x \in$ $B_{\delta_{x_{i}}}\left(x_{i}\right)$. We then get with (121) and (122) that

$$
\begin{aligned}
& \frac{2 \beta}{(\beta+1)^{2}} \int_{\Omega}\left|\nabla\left(\eta H_{L}(u)\right)\right|^{2} d x \\
& \leq \frac{4 \beta}{(\beta+1)^{2}} \int_{\Omega}|\eta \Delta \eta|\left|H_{L}(u)\right|^{2} d x+\int_{\Omega}\left|\Delta \eta^{2}\right| \cdot\left|J_{L}(u)\right| d x .
\end{aligned}
$$

Recall that it follows from Sobolev's inequality that there exists $K(n, 2)>0$ that depends only on $n$ such that

$$
\left(\int_{\mathbb{R}^{n}}|f|^{\frac{2 n}{n-2}} d x\right)^{\frac{n-2}{n}} \leq K(n, 2) \int_{\mathbb{R}^{n}}|\nabla f|^{2} d x
$$

for all $f \in H_{1,0}^{2}\left(\mathbb{R}^{n}\right)$. It follows from (123) and (124) that

$$
\begin{aligned}
& \frac{2 \beta}{(\beta+1)^{2}} K(n, 2)^{-1}\left(\int_{\Omega}\left|\eta H_{L}(u)\right|^{\frac{2 n}{n-2}} d x\right)^{\frac{n-2}{n}} \\
& \leq \frac{4 \beta}{(\beta+1)^{2}} \int_{\Omega}|\eta \Delta \eta|\left|H_{L}(u)\right|^{2} d x+\int_{\Omega}\left|\Delta \eta^{2}\right| \cdot\left|J_{L}(u)\right| d x
\end{aligned}
$$

for all $L>0$. As easily checked, there exists $C_{0}>0$ such that $\left|J_{L}(t)\right| \leq C_{0} \cdot|t|^{\beta+1}$ for all $t \in \mathbb{R}$ and all $L>0$. Since $u \in L^{\beta+1}(\Omega)$, we get that there exists a constant $C=C(\eta, u, \beta, \Omega)$ independant of $L$ such that

$$
\left.\int_{\Omega \cap B_{\delta_{x_{i}}}\left(x_{i}\right)}\left|H_{L}(u)\right|^{\frac{2 n}{n-2}} d x \leq \int_{\Omega} \mid \eta H_{L}(u)\right)\left.\right|^{\frac{2 n}{n-2}} d x \leq C
$$

for all $L>0$. Letting $L \rightarrow+\infty$, we get that

$$
\int_{\Omega \cap B_{\delta_{x_{i}}}\left(x_{i}\right)}|u|^{\frac{n}{n-2}(\beta+1)} d x<+\infty,
$$

for all $i=1 \ldots N$. We then get that $u \in L^{\frac{n}{n-2}(\beta+1)}(\Omega)=L^{\frac{n}{n-2} p}(\Omega)$. And then, $\frac{n}{n-2} p \leq p_{0}$ for all $p \in\left(2, p_{0}\right)$. Letting $p \rightarrow p_{0}$, we get a contradiction. Then $p_{0}=+\infty$ and $u \in L^{p}(\Omega)$ for all $p \geq 1$. This ends Step 8.2.

Step 8.3: We claim that

$$
u \in C^{0, \alpha}(\bar{\Omega})
$$

for all $\alpha \in(0,1)$. Indeed, it follows from Step 8.2 and the assumption $0<s<2$ that there exists $p>\frac{n}{2}$ such that

$$
f_{\epsilon}:=\frac{|u|^{2^{\star}-2-\epsilon} u}{|x|^{s}}-a u \in L^{p}(\Omega) .
$$

It follows from standard elliptic theory that, in this case, $u \in C^{0, \alpha}(\bar{\Omega})$ for all $\alpha \in(0, \min \{2-s, 1\})$. We let

$$
\alpha_{0}=\sup \left\{\alpha \in(0,1) / u \in C^{0, \alpha}(\bar{\Omega})\right\} .
$$


We let $\alpha \in\left(0, \alpha_{0}\right)$. Then $u \in C^{0, \alpha}(\bar{\Omega})$. Since $u(0)=0$, we then get that

$$
|u(x)| \leq|u(x)-u(0)| \leq C|x|^{\alpha} .
$$

We then get with (125) that

$$
\left|f_{\epsilon}(x)\right|=\left|\frac{|u(x)|^{2^{\star}-1-\epsilon} u}{|x|^{s}}-a u\right| \leq \frac{C}{|x|^{s-\left(2^{\star}-1-\epsilon\right) \alpha}}
$$

for all $x \in \Omega$. We distinguish 2 cases:

Case 8.3.1: $s-\left(2^{\star}-1-\epsilon\right) \alpha_{0} \leq 0$. In this case, for any $p>1$, up to taking $\alpha$ close enough to $\alpha_{0}$, we get that

$$
f_{\epsilon} \in L^{p}(\Omega) .
$$

Since $\Delta u+a u=f_{\epsilon}$ and $u \in H_{1,0}^{2}(\Omega)$, it follows from standard elliptic theory that there exist exists $\theta \in(0,1)$ such that $u \in C^{1, \theta}(\bar{\Omega})$. It follows that $\alpha_{0}=1$. This proves the claim in Case 8.3.1.

Case 8.3.2: $s-\left(2^{\star}-1-\epsilon\right) \alpha_{0}>0$. In this case, for any $p<\frac{n}{s-\left(2^{\star}-1-\epsilon\right) \alpha_{0}}$, up to taking $\alpha$ close enough to $\alpha_{0}$, we get that

$$
f_{\epsilon} \in L^{p}(\Omega) .
$$

We distinguish 3 subcases.

Case 8.3.2.1: $s-\left(2^{\star}-1-\epsilon\right) \alpha_{0}<1$. In this case, up to taking $\alpha$ close enough to $\alpha_{0}$, there exists $p>n$ such that

$$
f_{\epsilon} \in L^{p}(\Omega) .
$$

Since $\Delta u=f_{\epsilon}$ and $u \in H_{1,0}^{2}(\Omega)$, it follows from standard elliptic theory that there exist exists $\theta \in(0,1)$ such that $u \in C^{1, \theta}(\bar{\Omega})$. It follows that $\alpha_{0}=1$. This proves the claim in Case 8.3.2.1.

Case 8.3.2.2: $s-\left(2^{\star}-1-\epsilon\right) \alpha_{0}=1$. In this case, for any $p<n$, up to taking $\alpha$ close enough to $\alpha_{0}$, we get that

$$
f_{\epsilon} \in L^{p}(\Omega) .
$$

Since $\Delta u+a u=f_{\epsilon}$ and $u \in H_{1,0}^{2}(\Omega)$, it follows from standard elliptic theory that $u \in C^{0, \tilde{\alpha}}(\bar{\Omega})$ for all $\tilde{\alpha} \in(0,1)$. It follows that $\alpha_{0}=1$. This proves the claim in Case 8.3.2.2.

Case 8.3.2.3: $s-\left(2^{\star}-1-\epsilon\right) \alpha_{0}>1$. In this case, it follows from standard elliptic theory that $u \in C^{0, \tilde{\alpha}}(\bar{\Omega})$ for all

$$
\tilde{\alpha} \leq 2-\left(s-\left(2^{\star}-1-\epsilon\right) \alpha_{0}\right) .
$$

It follows from the definition of $\alpha_{0}$ that

$$
\alpha_{0} \geq 2-\left(s-\left(2^{\star}-1-\epsilon\right) \alpha_{0}\right)
$$

and then

$$
0 \geq 2-s+\left(2^{\star}-2-\epsilon\right) \alpha_{0}>0
$$

a contradiction since $s<2$ and $\epsilon<2^{\star}-2$. This proves that Case 7.3.2.3 does not occur, and we are back to the other cases.

Clearly, theses cases end Step 8.3.

Step 8.4: We claim that there exists $\theta \in(0,1)$ such that

$$
u \in C^{1, \theta}(\bar{\Omega}) \text {. }
$$


We proceed as in Step 8.3. We let $\alpha \in(0,1)$ (note that $\alpha_{0}=1$ ). We then get that

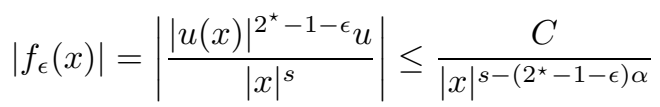

for all $x \in \Omega$. We distinguish 2 cases:

Case 8.4.1: $s-\left(2^{\star}-1-\epsilon\right) \leq 0$. In this case, for any $p>1$, up to taking $\alpha$ close enough to $\alpha_{0}$, we get that

$$
f_{\epsilon} \in L^{p}(\Omega) .
$$

Since $\Delta u+a u=f_{\epsilon}$ and $u \in H_{1,0}^{2}(\Omega)$, it follows from standard elliptic theory that there exist exists $\theta \in(0,1)$ such that $u \in C^{1, \theta}(\bar{\Omega})$. It follows that $\alpha_{0}=1$. This proves the claim in Case 8.4.1.

Case 8.4.2: $s-\left(2^{\star}-1-\epsilon\right)>0$. In this case, for any $p<\frac{n}{s-\left(2^{\star}-1-\epsilon\right)}$, up to taking $\alpha$ close enough to 1 , we get that

$$
f_{\epsilon} \in L^{p}(\Omega) .
$$

As easily checked,

$$
1-\left(s-\left(2^{\star}-1-\epsilon\right)\right)=2-s+\left(2^{\star}-1-\epsilon\right)-1>2^{\star}-2-\epsilon .
$$

We the get that there exists $p>n$ such that $f_{\epsilon} \in L^{p}(\Omega)$. Since $\Delta u+a u=f_{\epsilon}$ and $u \in H_{1,0}^{2}(\Omega)$, it follows from standard elliptic theory that there exists $\theta \in(0,1)$ such that $u \in C^{1, \theta}(\bar{\Omega})$. This proves the claim in Case 8.4.2.

Combining Case 8.4.1 and Case 8.4.2, we obtain Step 8.4. Proposition 8.1 then follows from Step 8.4.

\section{REFERENCES}

[1] Atkinson, F.V.; Peletier, L.A. Elliptic equations with nearly critical growth. J. Diff. Equ., 70, 349-365, 1987.

[2] Berestycki, H.; Nirenberg, L.; Varadhan, S.R.S. The principal eigenvalue and maximum principle for second order elliptic operators in general domains. Comm. Pure Appl. Math., 47, 47-92, 1994.

[3] Brézis, H.; Peletier, L.A. Asymptotics for elliptic equations involving critical Sobolev exponent. In Partial Differential equations and the calculus of variations, eds. F.Colombini, A.Marino, L.Modica, and S.Spagnolo, Birkhaüser, Basel, 1989.

[4] Caffarelli, L.; Gidas, B.; Spruck, J. Asymptotic symmetry and local behavior of semilinear elliptic equations with critical Sobolev growth. Comm. Pure Appl. Math., 42, (1989), 271-297.

[5] Caffarelli, L.; Kohn R. ; Nirenberg, L. First order interpolation inequality with weights, Compositio. Math. 53 (1984), 259-275

[6] Catrina, F.; Wang, Z. On the Caffarelli-Kohn-Nirenberg inequalities: sharp constants, existence (and non existence) and symmetry of extremal functions, Comm. Pure Appl. Math 2 (2001) 229-258.

[7] Druet, O. The best constants problem in Sobolev inequalities. Math. Ann., 314, 1999, 327346.

[8] Druet, O. Elliptic equations with critical Sobolev exponent in dimension 3. Ann. I.H.P., Analyse non-linaire, 19, 2, 2002, 125-142.

[9] Druet, O. From one bubble to several bubbles : the low-dimensional case. Journal of Differential Geometry, 63, 2003, 399-473.

[10] Druet, O.; Hebey, E.; Robert, F. Blow up theory for elliptic PDE's in Riemannian geometry. Mathematical Notes, Princeton University Press, 45. Announcement in A C0-theory for the blow up of second order elliptic equations of critical Sobolev growth. E.R.A./A.M.S., 9, 2003.

[11] Druet, O.; Robert, F. Asymptotic profile for the sub-extremals of the sharp Sobolev inequality on the sphere. Communications in Partial Differential Equations, 26, (2001), 743-778. 
[12] Egnell, H., Positive solutions of semilinear equations in cones, Tran. Amer. Math. Soc 11 (1992), 191-201.

[13] I. Ekeland, N. Ghoussoub, Selected new aspects of the calculus of variations in the large, Bull. Amer. Math. Soc. (N.S.) 39 (2002), no. 2, 207-265

[14] Gallot, S.; Hulin D.; and Lafontaine, J. Riemannian geometry, Springer-Verlag, 1987

[15] Ghoussoub, N.; Kang X.S. Hardy-Sobolev Critical Elliptic Equations with Boundary Singularities, AIHP-Analyse non linéaire, Vol 21 (2004) p. 767-793

[16] Ghoussoub, N.; Robert, F. Concentration estimates for a borderline equation with a singular potential on the boundary, 2005, submitted.

[17] Ghoussoub, N.; Robert, F. Concentration estimates for Emden-Fowler equations with boundary singularities and critical growth, 2005, submitted.

[18] Ghoussoub, N.; Yuan, C. Multiple solutions for quasi-linear PDEs involving the critical Sobolev and Hardy exponents, Trans. Amer. Math. Soc. 12 (2000), 5703-5743.

[19] Gidas, B.; Ni, W.M.; Nirenberg, L. Symmetry and related properties via the maximum principle. Comm. Math. Phys., 68, (1979), 209-243.

[20] Gilbarg, G.; Trudinger, N.,S. Elliptic partial differential equations of second order. Second edition. Grundlehren der mathematischen nWissenschaften, 224, Springer, Berlin, 1983.

[21] Han, Z.C. Asymptotic approach to singular solutions for nonlinear elliptic equations involving critical Sobolev exponent. Ann. Inst. H.Poincaré. Anal. Non Linéaire, 8, 159-174, 1991.

[22] Hebey, E. Asymptotics for some quasilinear elliptic equations. Differential Integral Equations, 9, (1996), no. 1, 71-88.

[23] Hebey, E.; Vaugon, M. The best constant problem in the Sobolev embedding theorem for complete Riemannian manifolds. Duke Math. J., 79, 235-279, 1995.

[24] Hebey, E.; Vaugon, M. From best constants to critical functions. Math. Z., 237, 737-767.

[25] Robert, F. Asymptotic behaviour of a nonlinear elliptic equation with critical Sobolev exponent. The radial case. Advances in Differential Equations, 6, (2001), 821-846.

[26] Robert, F. Critical functions and optimal Sobolev inequalities. Math. Z., 249, (2005), 485-492.

[27] Struwe, M. Variational methods, Springer-Verlag, Berlin-Heidelberg-New York, 1990.

[28] Schoen, R. Variational theory for the total scalar curvature functional for Riemannian metrics and related topics, in Topics in calculus of variations (Montecatini Terme, 1987). Lecture Notes in Mathematics, 1365, Springer, Berlin, 1989, 120-154.

Nassif Ghoussoub, Department of Mathematics, University of British Columbia, VanCOUVER, CANADA

E-mail address: nassif@math.ubc.ca

Frédéric Robert, Laboratoire J.A.Dieudonné, Université de Nice Sophia-Antipolis, Parc Valrose, 06108 Nice Cedex 2, France

E-mail address: frobert@math.unice.fr 